\title{
A T-splines-based Parametric Modeller for Computer-Aided Ship Design
}

\author{
T. Katsoulis, X. Wang, P.D. Kaklis ${ }^{1}$ \\ University of Strathclyde \\ Department of Naval Architecture, Ocean and Marine Engineering (NAOME) \\ Henry Dyer Building, 100 Montrose Street, Glasgow G4 OLZ, United Kingdom
}

\begin{abstract}
We present a T-splines-based parametric modeller (TshipPM) for complex ship forms, capable to provide smooth geometries at a low cost in comparison with parametric modellers (PM) employing the standard NURBS representation. For a given set of design parameters, we measure complexity via the number of degrees of freedom needed, i.e., the number of control points for representing the geometry of each instance. TshipPM, presented here, is an improved version of that in [1, enabling more flexibility for representing challenging areas of the ship-hull geometry, such as bow, stern and the transition areas from mid-ship towards forward and afterward perpendiculars. In this connection, the associated control-cage construction process, which maps the user-defined parameters to the control points of the T-splines representation, is described in detail for the forward transition part of the hull. Furthermore, TshipPM delivers instances lying in the proximity of a parent hull, with deviation measured in terms of moments (volume, volume centroid, moment of inertia) and sectional area curve (SAC) distribution, which are used in ship design. Finally, TshipPM is compared against a commercial PM, CAESES ${ }^{\circledR}$, opting for its NURBS functionality, with both PMs' outputs compared against a parent container-ship hull already used in the literature for CAD and CFD benchmarking purposes. The employed comparison criteria include the common external parameters,
\end{abstract}

\footnotetext{
${ }^{1}$ corresponding author: Panagiotis Kaklis, email address: panagiotis.kaklis@strath.ac.uk
} 
the previously mentioned moments, the sectional area curve, Gaussian and sectional curvatures for assessing surface and curve fairness, respectively, and the Hausdorff distance for measuring the geometric distance between two hulls. Keywords: CAD, Computer-Aided Ship Design, Parametric Modelling; NURBS, T-splines

\section{Introduction}

Parametric Modellers (PM) play a crucial role in shape optimisation of engineering systems, for they have to represent robustly and efficiently every solid object. In general, a PM is an algorithmic scheme depending on a set of geo5 metric parameters referred to as external parameters, accessible to the user, the number of which determines the dimension of the design space associated with the given PM. Attributing values to all members of this set, PM is expected to deliver an acceptable instance of the shape to be optimised. Acceptability is tested against the fundamental requirement that the bounding surface of the generated object should be free from self-intersections. Furthermore, various application-stemming requirements have to be met, governed by shape descriptors selected for evaluating the quality of the generated instance. Typical examples of such descriptors are the parametric or geometric continuity, the fairness, i.e., non-oscillatory distribution of intrinsic geometric features, such

15 as curvature, torsion, Gaussian curvature, and integral characteristics, such as moments of various orders, e.g., volume, centroids, moments of inertia.

Adopting the timeline introduced by Prof. H. Nowacki [2, who pioneered in Computer-Aided Ship Design (CASD) via coupling form parameters with the then novel technology of B-splines [3, the research and user communities will 20 celebrate on 2020 the key developments of CASD since its inception about six decades ago. An early attempt for building a ship parametric model is due to Lackenby 4 in which hull variants are obtained by modifying the prismatic coefficient, the center of buoyancy and the extent and position of the cylindrical mid-body of a parent hull. This approach has been subsequently 
generalised towards expanding the geometric coverage and portability of the parametric model through the use of NURBS (Non-uniform rational B-splines), which is the CAD (Computer-Aided Design) industrial standard for designing and processing curves and surfaces; see, e.g., [5], 6], [7, [8] and [9]. As a result, a number of PMs are currently available to ship-design practitionso ers, such as CAESES $\AA^{\circledR 2}$, CATIA ${ }^{\circledR} 3$, the Rhino3D-based GRASSHOPPER $\AA^{\circledR} 4$ MARIN/GMS ${ }^{\circledR 5}$, NAPA ${ }^{\circledR}$ [ and PARAMARINE ${ }^{\circledR 7}$.

NURBS-based technology is not however free of shortcomings when applied to parametric modelling of complex ship forms involving many and functionally critical regions of shape transition nature; see, e.g., [10]. One can attribute these limitations to the underlying tensor-product character of NURBS, which implies the need for building smoothly connected (at least $G^{1}$-continuous) NURBS patches over areas with different shape characteristics (convex, flat, cylindrical, saddle) and varying scale. As a result, it is not unlikely that designers and engineers have to deal with highly complex NURBS models, involving large before feeding them to the solver and optimiser for shape optimisation. In order to handle the challenge of seamless integration between parametric modelling and shape optimisation, more generally, integrating CAD with CAE (ComputerAided Engineering), several alternative representations have been developed in 45 pertinent literature, including hierarchical splines [11], PHT-splines [12], LRsplines [13] and T-splines [14, 15, [16]. T-splines constitute a generalisation of NURBS technology that removes several of NURBS deficiencies, e.g., enabling refinement without the need of adding redundant control points. Furthermore, they have been successfully used in conjunction with Isogeometric

\footnotetext{
${ }^{2}$ https://www.caeses.com/

${ }^{3}$ https://www.3ds.com/

${ }^{4}$ https://www.grasshopper3d.com/

${ }^{5}$ http://www.marin.nl/

${ }^{6}$ https://www.napa.fi/

${ }^{7}$ https://paramarine.qinetiq.com/
} 
Analysis (IGA) in several areas of computational mechanics; see e.g., [1], [17], [18], 19], 20], 21], 22], 23]. IGA is a new methodology that eliminates the need of geometry discretisation (meshing), as it is the case for standard FEM (Finite Element Methods) and BEM (Boundary Element Method), enabling a seamless and strong coupling between the geometry representation and the solver 8

Regarding parametric modelling, T-splines technology is capable to develop modellers for complex ship forms, which provide acceptable and smooth (at least) $G^{1}-$ continuous geometries at the expense of lower complexity in comparison with PMs employing the standard NURBS-based technology. For a given set of design parameters and their ranges, we measure complexity via the number of DoF (Degrees of Freedom) required for representing the geometry of each instance, i.e., the number of control points involved in the two competing representations. Since T-splines achieve to span the same design space with considerable less DoF than NURBS, it is legitimate to assert that their efficiency, expressed as the ratio of shape-richness over DoF, is higher than that of NURBS. Furthermore, it is worth noticing that DoF downsizing is beneficial in the subsequent steps of shape optimisation, e.g., by decreasing analogously the size of stiffness matrices involved in the adopted FEM/BEM solver.

In this paper we present and test TshipPM, an in-house developed PM for ship design. TshipPM is based on T-splines functionality provided by Rhino3D ${ }^{\circledR}$ scripting language and Autodesk ${ }^{\circledR}$ T-splines plug-in. Our work extends that in [1] along four directions:

- We provide the reader with the list of all physical (dimensional) parameters that characterise the geometry of the instances created by TshipPM along with a concise description of each one of them, their classification in

\footnotetext{
${ }^{8}$ The reader interested in the theoretical foundations and research on the analytical properties of T-splines, e.g., dimension of T-splines spaces, linear independence of T-splines basis functions, can appeal to an abundance of pertinent papers in the area of Computer-Aided Geometric Design; see, e.g., 24], 25], 26].
} 
global and non-global categories and, finally, their functional interrelation, which also involves the non-dimensional parameters; see $§ 3.1$.

- TshipPM enables a more flexible representation of ship hull in geometrically challenging areas, namely bow, stern and the transition areas from mid-ship towards the forward and afterward perpendiculars; see $§ 3.2$.

- The paper illustrates a key feature of the control-cage construction process, namely the mapping of the external parameters to the control points of the T-splines representation, by describing it in detail for the forward transition part of the hull; see $§ 3.2$.

- TshipPM is improved to deliver instances lying in the proximity of a parent hull with deviation measured in terms of moments (volume, volume centroid, moment of inertia) and sectional area curve (SAC), which are being used by ship designers; see $§ 3.3$.

In addition, TshipPM is compared against the commercial CAESES PM, by comparing their outputs against a parent hull, the KCS container-ship hull [27, which has been extensively used by the research community for CAD and CFD benchmarking purposes. The employed comparison includes the common external parameters, the sectional area curve, the Gaussian and sectional curvatures for assessing surface and curve fairness, respectively, and, lastly, Hausdorff distance; see $\S 4$.

The rest of the paper is structured in four sections. The ensuing section (§2) delivers a coarse comparison between T-splines, the representation underlying TshipPM, and NURBS, used in the context of CAESES. Section 3 analyses the structure of TshipPM regarding the parameters involved (§3.1), the building of the control cage along with its link with the parameters and the resulting surface (§3.2), while $\S 3.3$ illustrates the robustness of TshipPM versus strong parameter variations. Section 4, devoted to the comparison of TshipPM with CAESES, consists of seven subsections: Firstly, a short introduction to CAESES PM (§4.1) followed by a presentation of the external parameters which are common 
NURBS is the current geometry standard for representing curves, surfaces and, to some extent, solids in CAD systems. T-splines is a generalisation of NURBS representation for surfaces and solids with their main advantage over NURBS being to allow a row of control points to terminate before reaching the boundary, configuration in T-splines is called a T-junction. The locally rectangular control mesh, where T-junctions exist, is called a T-mesh 14 . A control point $\mathbf{P}_{A} \in \mathbb{R}^{3}$, and a control weight $w_{A}>0$ are assigned to every vertex of the T-mesh, where the index $A$ denotes a global control-point counter, $A=0,1,2, . ., n, n \in \mathbb{N}$.

Focusing on odd-degree splines, which possess the "well known" minimum pseudonorm property (in engineering terms: linearised-elastic-energy minimisers), each member of a T-splines basis can be associated in a one-to-one manner with each of the control points. Assuming that $R_{\mathrm{A}, \mathrm{p}}(s, t)$ represents the $A^{\text {th }}$ polynomialspline basis function of bi-degree $p=2 \rho+1, \rho=1,2, \ldots$, for surfaces, the resulting T-splines surface can be represented as:

$$
\mathbf{c}(s, t)=\sum_{A=0}^{n} \mathbf{P}_{A} N_{A, p}(s, t),(s, t) \in \Omega \subset \mathbb{R}^{2},
$$

where

$$
N_{A, p}(s, t)=\frac{w_{A} R_{A, p}(s, t)}{\sum_{J=0}^{n} w_{J} R_{J, p}(s, t)} .
$$

T-splines bases inherit the basic properties of NURBS bases as summarised below: 
(i) partition of unity: $\sum_{i=1}^{n} N_{\mathrm{A}, \mathrm{p}}(s, t)=1$ with $(s, t)$ varying in a subdomain $\omega$ of the global domain of definition $\Omega \subset \mathbb{R}^{2}$;

(ii) non-negativity: $N_{\mathrm{A}, \mathrm{p}}(s, t) \geq 0$ with $(s, t) \in \mathbb{R}^{2}$;

125

(iii) compact support:

$N_{\mathrm{A}, \mathrm{p}}(s, t)=0$ if $(s, t) \notin\left[s_{A-(\rho+1)}, s_{A+(\rho+1)}\right] \times\left[t_{A-(\rho+1)}, t_{A+(\rho+1)}\right]$

( $\rho=1$ for the typical bicubic case);

(iv) continuity: in the neighbourhood of a knot line of multiplicity $k, N_{\mathrm{A}, \mathrm{p}}(s, t)$ is $C^{p-k}$ continuous;

(v) convex-hull property: a T-splines $\sum_{A=0}^{n} \mathbf{P}_{\mathrm{A}} N_{\mathrm{A}}(s, t),(s, t) \in \tilde{\omega} \subset \mathbb{R}^{2}$, lies within the convex hull of the subset of control points $\mathbf{P}_{A}, i=1, \ldots, n$, for which the compact support of the corresponding basis functions intersects $\tilde{\omega}$;

(vi) affine invariance: a $\mathrm{T}$-splines is invariant with respect to affine transformations;

(vii) boundary-curve interpolation, via allocating appropriate multiplicity at the boundary knots, and

(viii) linear independence for a topologically restricted subset of T-splines, referred to as analysis-suitable T-splines, optimised to meet the needs for both design and analysis; see, e.g., [28]).

While in NURBS all basis functions are defined via the tensor product of two 1D global knot vectors, in T-splines a local knot vector is assigned to every basis function, with each local knot vector having an association with the topology of the whole patchwork of the object [19]. This difference enables T-splines to permit T-junctions, while for the same refinement (knot insertion) shorter knot lines are generated. Fig. 1 illustrates the fundamental difference between a NURBS mesh and a T-mesh. In this paper, due to the fact that it suffices for the needs of our application to work with $G^{1}$-continuous surfaces, we shall limit our discussion to bicubic T-splines.

${ }^{150}$ It is well known from CAD practice that NURBS need multiple patches to represent complex shapes, complicating the analysis and optimisation of the 

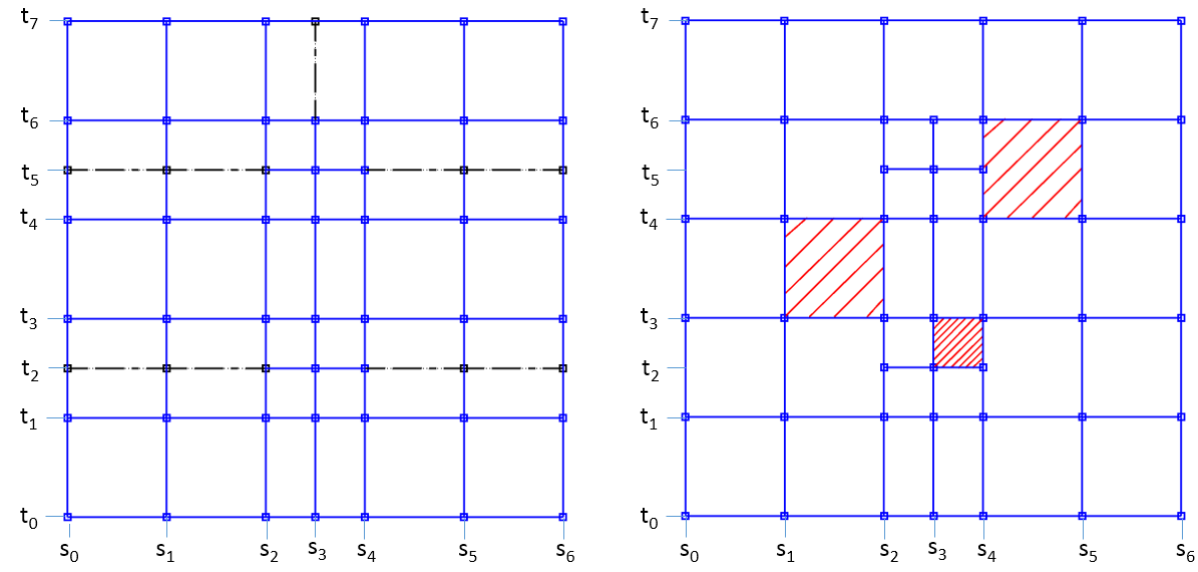

Figure 1: NURBS (left) knot lines lie on a global rectangular grid, while T-splines (right) can form T-junctions due to locally defined knot vectors. Extraneous knot lines in NURBS are depicted with black dotted line. Examples of parametric faces depicted in red diagonal stripe pattern: one face (rightmost) with 5 vertices (four corner vertices and one T-junction vertex) and two faces with four corner vertices. Note that all faces, in both the NURBS and the T-splines mesh, are rectangular.

design. On the contrary, it is not unlikely that T-splines are capable to represent the same shapes with a single patch. In addition, refinement with NURBS generates superfluous control points, as NURBS must lie topologically on a rectangular grid, which is not the case in T-splines due to the fact that they permit T-junctions. Although, T-splines are not totally free of superfluous control points. Furthermore, in various cases, trimming of NURBS surfaces cannot be avoided. A curve stemmed by trimming is generally not a NURBS curve, therefore approximation is needed to represent it. On the other hand, T-splines are able to represent such surfaces without trimming with the aid of T-junctions 29]. In addition, T-splines can produce a valid merging of multiple NURBS patches into a watertight surface without gaps, see, e.g., Fig. 3 in [14]. Given a T-mesh in the parametric space and a valid knot-interval configuration, we describe the process of creating the T-splines bicubic basis corresponding, e.g., to the vertex $\mathbf{V}_{0}$ depicted in Fig. 2, as follows:

(i) Starting from the vertex $\mathbf{V}_{0}$, the horizontal knot vector is obtained by 
marching towards each of the two horizontal directions until two sequential edges or vertices are encountered. The corresponding univariate basis is the cubic B-spline $N_{0,3}(s)$ defined on the local knot vector $\left\{s_{1}, s_{2}, s_{3}, s_{4}, s_{5}\right\}$ :

$$
N_{0,3}(s) \equiv N\left[s_{1}, s_{2}, \mathbf{s}_{\mathbf{3}}, s_{4}, s_{5}\right](s)
$$

(ii) Create in directly analogous manner the univariate basis along the vertical direction.

$$
N_{0,3}(t) \equiv N\left[t_{3}, t_{4}, \mathbf{t}_{\mathbf{5}}, t_{6}, t_{7}\right](t)
$$

(iii) The product $N_{0,3}(s) N_{0,3}(t)$ of the two univariate basis functions gives the sought-for T-splines bicubic basis function corresponding to $\mathbf{V}_{0}$.

Note that a vertex in the parametric space corresponds to a specific control point of the T-mesh in the physical space.
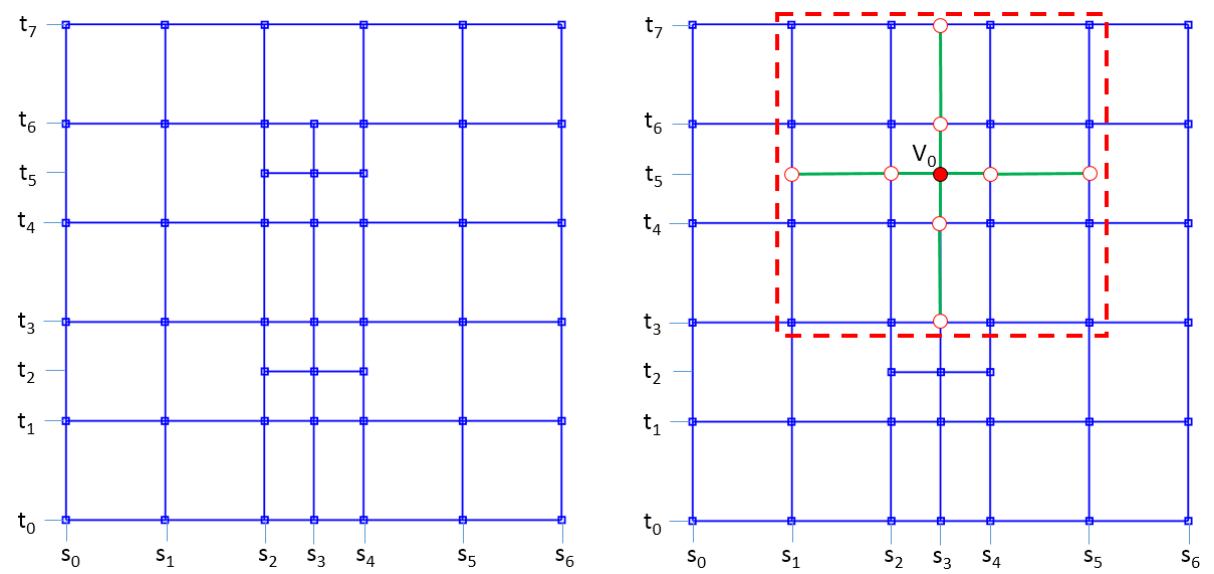

Figure 2: Constructing the T-splines basis function corresponding to the vertex $\mathbf{V}_{0}$ of a valid T-mesh. The extents of the constituting univariate knot vectors are colored green, while the boundary of the support of the resulting basis function is depicted as dashed polygonal line colored red.

\section{Parametric Modelling using T-splines}

In this section we outline the construction of a T-splines-based PM for ship hull design, referred to as TshipPM. After a short introduction on the philosophy 
adopted for building the modeller, the section is structured in three subsections. Subsection 3.1 introduces the physical (dimensional) parameters along with a concise description of each one of them, their classification in global and non-global categories and, finally, their interrelation via the non-dimensional parameters. Subsection 3.2 is devoted to the process of defining the geometry and connectivity (topology) of the control cage underlying the T-spline surface. Additionally, we provide in detail for the forward transition part of the hull the mapping of the user-specified parameters to the control points of the control cage. Finally, we illustrate the full output of TshipPM, i.e., control cage and surface, for a container-ship hull and discuss the gains with respect to the required number of control points when using T-splines versus NURBS representation. The section ends with subsection $\$ 3.3$ illustrating the robustness of TshipPM via a set of geometrically valid instances resulting from strong parameters variations.

Our approach of constructing TshipPM is twofold: firstly to develop a PM that is considerably more flexible in representing ship hulls than that in [1] and secondly to provide instances lying in the proximity of a given parent ship hull in terms of ship-design criteria such as up to $2^{\text {nd }}$ order moments and SAC. For this purpose, TshipPM is equipped with the functionality to produce additional transition curves, enabling a finer control of the geometry construction from mid-ship towards forward (FP) and afterward (AP) perpendiculars, as well as a more accurate representation of bow and stern. The above enhancements are achieved at the expense of only three parameters in comparison to [1]. The number of control points increases by $45 \%$ (134 vs 196) in comparison to that in 1, nevertheless the order of magnitude remains the same, $O\left(10^{2}\right)$, i.e., well below than that required when using NURBS, $O\left(10^{3}\right)$.

\subsection{Parameters: introduction and handling}

${ }_{200}$ TshipPM has been developed using Rhinoceros ${ }^{\circledR}$ scripting functionality, based on Visual Basic ${ }^{\circledR}$, and employs Autodesk ${ }^{\circledR}$ T-splines plug-in ${ }^{\circledR}$ V.4.0 for Rhinoceros $5{ }^{\circledR}$ $3 \mathrm{D}$ to create T-splines surfaces using as input the control cage created by 
TshipPM. Rhinoceros5 ${ }^{\circledR} 3 \mathrm{D}$ is a broadly used CAD software based on NURBS for modelling curves, surfaces and solids. Autodesk plug-in adds T-splines functionality to Rhinoceros which with the support of Rhino Script generates T-splines models. In analogy to the control polygon of curves, the geometry and topology of the control cage influences decisively the shape of the underlying T-splines surface to be generated. It is a network of linear edges connecting the given control points, and in conjunction with the associated T-splines basis it delivers the shape of the surface.

TshipPM's main purpose is to generate the control cage of a ship hull with the aid of parameters of both external and internal character. It is currently relying on 27 physical parameters $P_{i}, i=0, \ldots, 26$, which characterise the geometry of the control cage under construction. These are dimensional quantities and are classified in global and non-global ones, the latter being further categorised in 5 groups, according to which part of the ship they belong; see Table 1. Besides the first three physical parameters: $P_{0}=\mathrm{Lwl}$ (length of the waterline), $P_{1}=\mathrm{B}$ (beam) and $P_{2}=\mathrm{T}$ (draft), the remaining physical parameters are defined according to the following scheme:

$$
P_{i}=\hat{P}_{i} \cdot f_{i}\left(P_{0}, . ., P_{i-1}\right), \quad \hat{P}_{i} \in(0,1), i=3, \ldots, 26,
$$

where $\hat{P}_{i}$ are the non-dimensional parameters and $f_{i}$ are affine functions of the physical parameters. In matrix form the above relations can be represented as:

$$
\mathbf{P}^{\prime}=\operatorname{diag}(\hat{P})(\mathbb{A} \mathbf{P}+\mathbf{v}), \quad \mathbf{P}^{\prime}=\left[P_{3}, \ldots, P_{26}\right]^{T}, \hat{\mathbf{P}}=\left[\hat{P}_{3}, \ldots, \hat{P}_{26}\right]^{T},
$$

where $\operatorname{diag}(\hat{P})$ denotes the diagonal matrix defined by the non-dimensional parameters $\hat{P}_{i}$ and $\mathbb{A}$ is a rectangular matrix of block-triangular structure. Both physical, $P_{i}$, and non-dimensional, $\hat{P}_{i}$, parameters are of external (user-visible) nature. On the contrary, internal parameters are accessible only to the developer and are deployed for shape stabilisation via retaining key shape characteristics of the type of the hull the designer is interested in. Their number varies, depending on the complexity and the number of control curves which identify challenging 
areas of shape transition between mid-ship and the bow/stern areas of the ship. Once parameters values are provided to the first three physical parameters and the non-dimensional parameters, Autodesk T-splines plug-in is called using as input the control cage created by TshipPM to produce the final T-splines model. Fig. 3 provides a representative diagram of the execution process.

\begin{tabular}{|c|c|c|c|c|c|c|c|}
\hline Input & $\begin{array}{c}\text { External } \\
+ \\
\text { Internal } \\
\text { Parameters }\end{array}$ & Construct & Control Cage & Employ & $\begin{array}{l}\text { T-splines } \\
\text { Plug-In }\end{array}$ & Output & $\begin{array}{l}\text { Ship-hull } \\
\text { Instance }\end{array}$ \\
\hline
\end{tabular}

Figure 3: Steps to create a TshipPM instance: we first attribute values to $P_{0}=\mathrm{Lwl}, P_{1}=\mathrm{B}$, $P_{2}=\mathrm{T}$ and the non-dimensional parameters $\hat{P}_{i} \in(0,1), i=3, \ldots 26$, which, alongside the internal parameters, determine the control cage of the ship hull; TshipPM plug-in is then called to produce the final instance.

Table 1: TshipPM parameters and groups; $P_{i}$ 's: physical parameters

\begin{tabular}{ccc}
\hline$P_{i}$ 's & Description & Affine functions $f_{i}$ of \\
\hline \hline$P_{0}=$ Lwl & Global Parameters \\
$P_{1}=\mathrm{B}$ & Beam & - \\
$P_{2}=\mathrm{T}$ & Draft & - \\
\hline \multicolumn{3}{c}{ non-Global Parameters } \\
\hline$P_{3}=$ Mid_L & Mid_Part Parameters \\
$P_{4}=$ Mid_Pos & Length of Middle Body & Lwl \\
& ongitudinal middle position & Lwl, Mid_L \\
$P_{5}=$ Bilge_R & Bilge Radius & B, T \\
\hline & Fwd_Part Parameters & B \\
$P_{6}=$ BatFP & Breadth at FP & Mid_Pos, Mid_L \\
$P_{7}=$ FoS_Fwd_L & Flat of Side & Mid_Pos, Mid_L \\
$P_{8}=$ FoB_Fwd_L & Flat of Bottom & Continued on next page
\end{tabular}


Table 1 - Continued from previous page

\begin{tabular}{ccc}
\hline$P_{i}$ 's & Description & Affine functions $f_{i}$ of \\
\hline$P_{9}=$ FoS_Trans & Flat of Side at the & $\mathrm{T}$ \\
& transition of MidShip & \\
& to Fwd $/$ Aft_Part & \\
$P_{10}=$ FoB_Trans & Flat of Bottom at the & $\mathrm{B}$ \\
& transition of Midship & \\
& to Fwd $/$ Aft_Part & \\
$P_{11}=$ FP_L_fromMS & Length from Mid Body to FP & Mid_Pos, Mid_L, \\
& & Fos_Fwd_L \\
\hline
\end{tabular}

Aft_Part Parameters

\begin{tabular}{ccc}
\hline$P_{12}=$ BatAP & Breadth at AP & B \\
$P_{13}=$ BatAPLow & Breadth at the bottom at AP & B \\
$P_{14}=$ FoS_Aft_L & Flat of Side & Lwl, Mid_Pos, Mid_L \\
$P_{15}=$ FoB_Aft_L & Flat of Bottom & Lwl, Mid_Pos, Mid_L \\
$P_{9}=$ FoS_Trans & Flat of Side at the & T \\
& transition of MidShip & \\
& to Fwd $/$ Aft_Part & B \\
$P_{10}=$ FoB_Trans & Flat of Bottom at the & \\
& transition of Midship & \\
$P_{16}=$ AP_L_FromMS & to Fwd $/$ Aft_Part & Lwl, Mid_Pos, \\
& & Mid_L, Fos_Aft_L \\
\hline$P_{17}=$ Fwd_Rise & Rise of Fwd_Part & Bulb_H \\
$P_{18}=$ Bulb_L & Bulbous Length (from FP) & Lwl \\
$P_{19}=$ Bulb_H & Bulbous Height & T \\
$P_{20}=$ Bulb_B & Bulbous Width & B \\
$P_{21}=$ Bulb_Tip_Height & Height of tip of Bulbous & Bulb_H \\
\hline
\end{tabular}

Continued on next page 
Table 1 - Continued from previous page

\begin{tabular}{ccc}
\hline \multicolumn{1}{c}{$P_{i}$ 's } & Description & Affine functions $f_{i}$ of \\
\hline$P_{22}=$ Transom_H & Stern Parameters & \\
$P_{23}=$ Transom_B & Transom Height & T \\
$P_{24}=$ Stern_L & Stern Length from AP & BatAP \\
& & Lwl, Mid_Pos, \\
& & Mid_L, FoS_Aft_L, \\
$P_{25}=$ Tube_L & Tube Length & AP_L_FromMS \\
$P_{26}=$ Tube_D & Tube Diameter & Transom_H \\
\hline
\end{tabular}

TshipPM favours the use of non-dimensional parameters, where possible, in order to handle effectively the interdependency that occurs among them, as it is the case in (4). For example, if we define ship's draft $T$ as a physical parameter the permissible value would be upper-bounded by the ship's depth $D$. On the other hand, by employing the ratio parameter: $r=\frac{T}{D}$, its valid range bounds are 0 and 1 regardless of ship's depth value, which permits a simpler checking algorithm for the validation of input parameter values. The benefits of this approach are more evident when we have a series of interdependent parameters, as in the case of ship's mid-ship part definition in the example below.

Let us consider a ship of length $l$, and two points defining the starting and terminating points of the mid-ship part, respectively. Furthermore, let $l_{S}$ and $l_{E}$ denote the corresponding distances of each of these points from the common origin O; see Fig. 4. If we were to implement $l_{S}$ and $l_{E}$ as physical parameters, we would have to include validation checks so that, for example, the inequality $0<l_{S}<l_{E}<l$ stands true, whereas, by introducing ratio parameters $r_{E}=\frac{l_{E}}{l}$ and $r_{S}=\frac{l_{S}}{l_{E}}$, we ensure the right order of the corresponding points by simply bounding both ratios in $(0,1)$; see Fig. 4. Obviously, in this case, no further validation checking is required. 


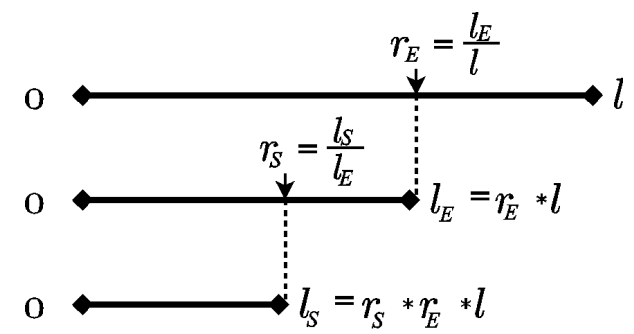

Figure 4: Relation between physical (dimensional) and non-dimensional parameters.

Thus, the use of non-dimensional parameters, ranging in subintervals of $(0,1)$, supports the robustness of TshipPM, by avoiding the allocation of values to parameters that would result in delivering non-valid geometries suffering e.g., from self-intersections. These subintervals are currently pre-specified by the developer via experimentation with the available parent hulls. Figs. 50 7 illustrate all physical parameters used in TshipPM. In the rest of the text the term $p a$ rameters will be used for referring only to the three physical and the twenty-four non-dimensional parameters, denoted by symbols with a wide hat symbol, e.g., $\widehat{B u l b \_} L$ denotes the non-dimensional parameter corresponding to the physical parameter of bulbous-bow length, Bulb_L .

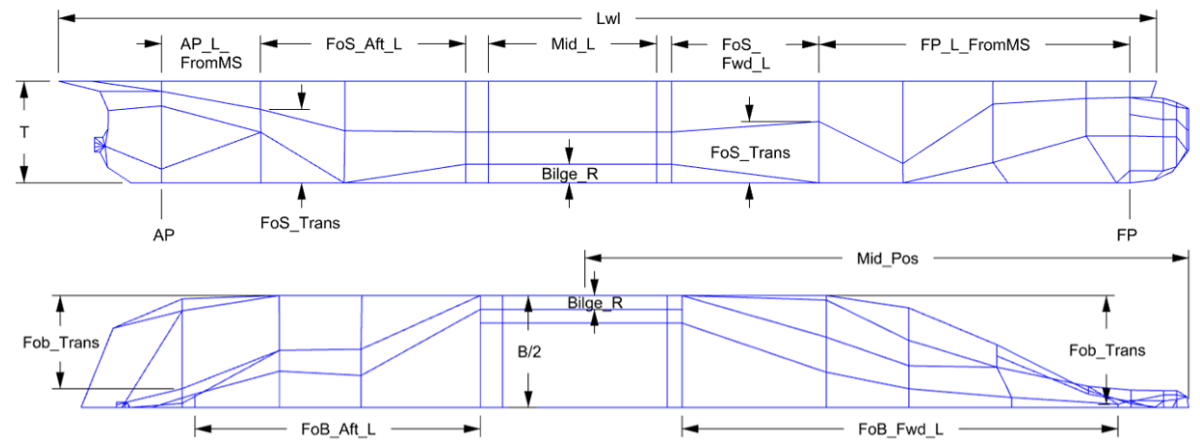

Figure 5: TshipPM input parameters for Fwd_Part and Aft_Part. 

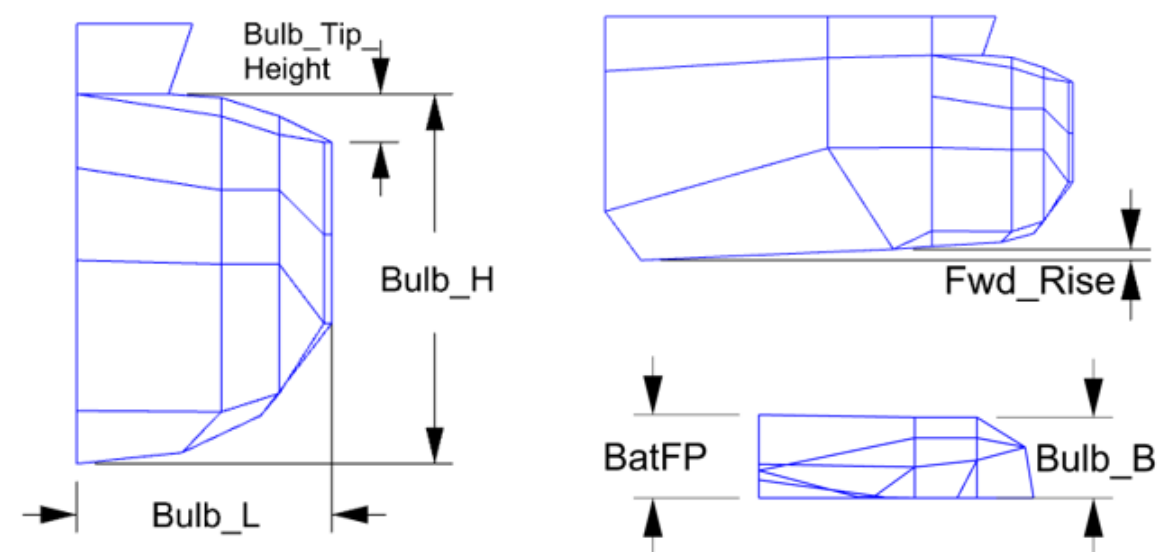

Figure 6: TshipPM Bulb input parameters.
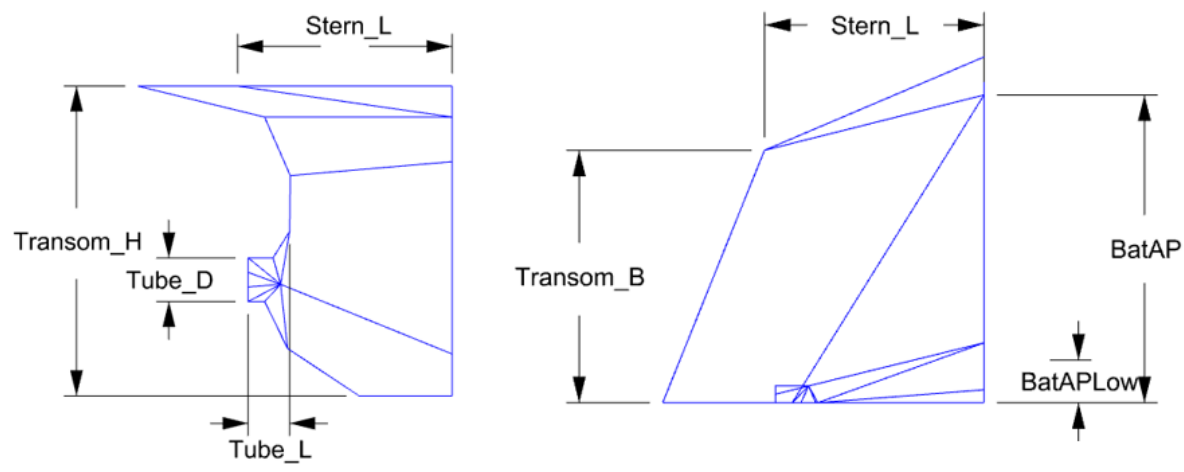

Figure 7: TshipPM Stern_L, BatAP and BatAPLow input parameters.

\subsection{From parameters to the control cage and the output surface}

a) the mid-ship, which is the part bounded by the FP and AP, b) the bow, which extends from FP to the forward end and c) the stern, extending from AP and afterwards. To describe adequately the main parts of a ship hull, global physical parameters are needed, such as Lwl, B and T, as well as mid-ship, consisting of the cylindrical middle part (Mid_Part), the forward (Fwd_Part) and the afterward (Aft_Part) parts. This multi-level segmentation of the ship hull to the aforementioned parts should be reflected on the control cage; see 
Figs. 8 and 9 .

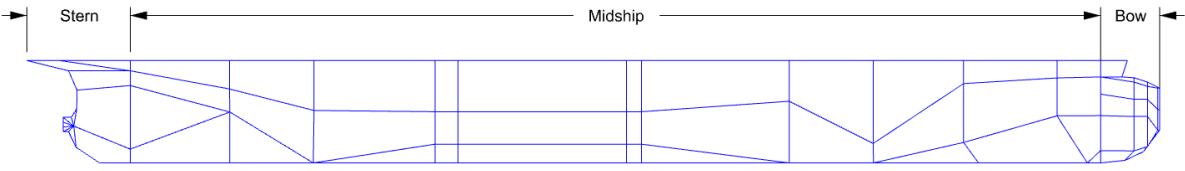

Figure 8: Typical segmentation of a ship hull. Main parts are mid-ship, bow, and stern.

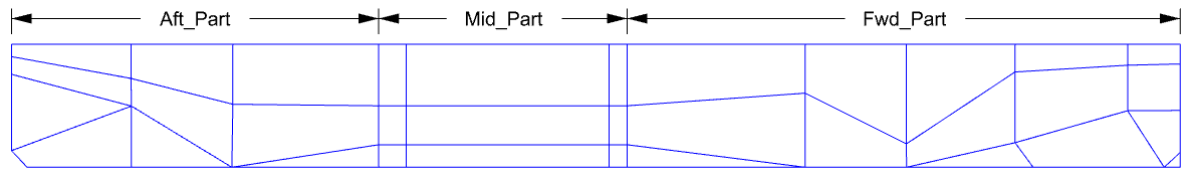

Figure 9: Segmentation of mid-ship, consisting of the Cylindrical Middle Part, Forward and Afterward Parts.

In order to give shape to the bounding surfaces of a ship hull and guarantee a fair transition from a part to its neighbouring ones, control curves are required. Control curves are of two types, namely, bounding curves and transition curves. Bounding curves, such as bow (Bow_Prf_Crv) and stern profile (Stern_Prf_Crv) curves, are used to specify the boundaries of the ship hull, while transition curves are employed to indicate the transition between different parts and/or further segmentation of the same part. Pre-images of these curves on the control-cage level are introduced by the so-called control-cage paths as illustrated in Fig. 10.
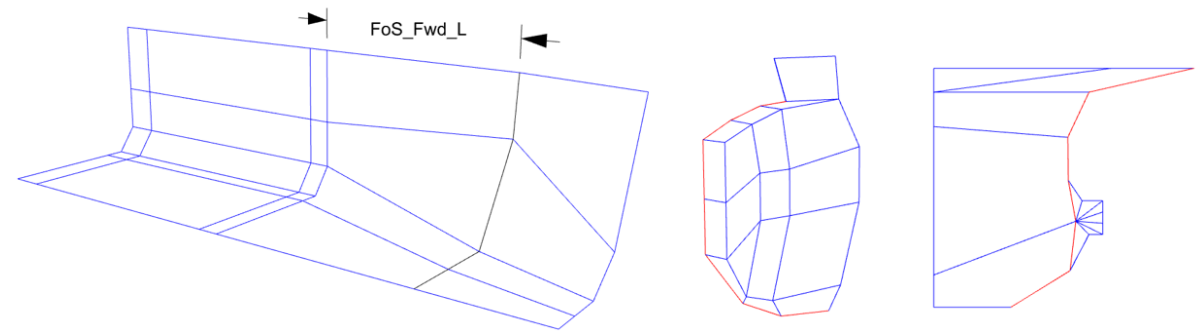

Figure 10: Examples of control-cage paths of transition type (left: from flat of side to forward part, depicted in black) or boundary type (middle: stern boundary, right: bow boundary, depicted in red). 
Transition from one part to another is materialised via external parameters. Determining control-cage paths, though, requires both internal and external parameters. An example is given in Fig. 11] where it is assumed that the extent of Mid_Part is already fixed. Then, the external parameter controlling the longitudinal transition from the end of Mid_Part to the boundary Fwd_Trans_Crv of FoS_Fwd_L, is parameter Fo $\widehat{S_{-} F w} d_{-} L$, while Fwd_Trans_Crv makes use of parameters FoS_Trans, Fob_Trans and the internal parameter $\alpha$.

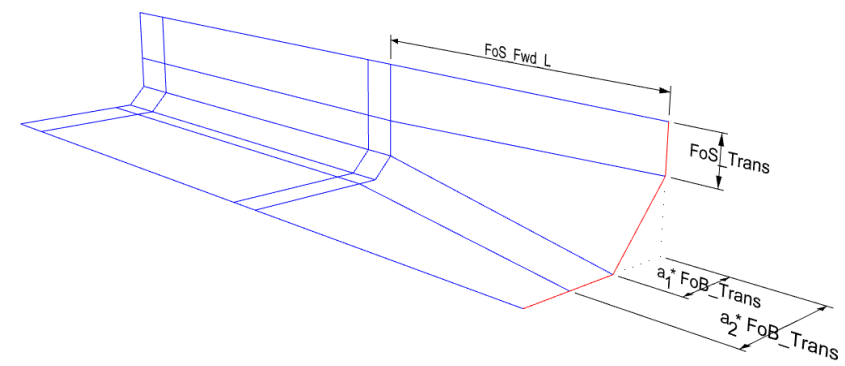

Figure 11: Control-cage path Fwd_Trans_Crv (red) and its defining parameters.

Let us now describe in more detail the process adopted by TshipPM for mapping user-defined parameters to the control points of the control cage for the configuration depicted in Fig. 12, associated with the challenging transition area from the mid-body towards FP. To proceed, we assume that the control points $\mathbf{M}_{1, i}=\left(M_{1, i}^{x}, M_{1, i}^{y}, M_{1, i}^{z}\right)^{T}, i=0 ., \ldots, 5$, of the L-shaped control polygon $\mathcal{M}_{1}$ (depicted in black) have been calculated, adopting the choices made in Fig. 6 in $§ 2$ of [1], and we aim to compute the control points of the first two Forward Transition Curves (four in total), namely $\mathcal{F} \mathcal{T} \mathcal{C}_{0}$ (depicted in red) with control points $\mathbf{P}_{0, \mathrm{i}}, i=0,1, . ., 5$ and $\mathcal{F} \mathcal{T} \mathcal{C}_{1}$ (depicted in green) with $\mathbf{P}_{1, \mathrm{i}}, i=0,1, . ., 5$. TshipPM calculates the location of the afore mentioned control points using the below formulae $(7)-(11)$ for $\mathcal{F} \mathcal{T C}_{0}$ and $(12)-(16)$ for $\mathcal{F} \mathcal{T} \mathcal{C}_{1}$. 


$$
\begin{aligned}
& \mathbf{P}_{0,0}=\mathbf{M}_{1,0}-\left(F_{o} S_{-} F w d_{-} L, 0,0\right)^{T}, \\
& \mathbf{P}_{0,1}=\mathbf{P}_{0,0}-\left(0,0, a_{0} \cdot \text { FoS_Trans }\right)^{T}, \\
& \mathbf{P}_{0,2}=\left(M_{1,0}^{x}-a_{1} \cdot \text { FoB_Fwd_L }, O^{y}-a_{2} \cdot \text { FoB_Trans, } O^{z}-T\right)^{T} \text {, } \\
& \mathbf{P}_{0,3}=\left(P_{0,2}^{x}, O^{y}-a_{3} \cdot \text { FoB_Trans, } P_{0,2}^{z}\right)^{T}, \\
& \mathbf{P}_{0,4}=\left(P_{0,3}^{x}, O^{y}-\frac{B}{2}, P_{0,3}^{z}\right)^{T} . \\
& \mathbf{P}_{1,0}=\mathbf{P}_{0,0}-\left(a_{4} \cdot \text { FP_L_From } M S, 0,0\right)^{T}, \\
& \mathbf{P}_{1,1}=\left(P_{1,0}^{x}, P_{1,0}^{y}-a_{5} \frac{B}{2}, O^{z}-a_{6} \cdot T\right)^{T}, \\
& \mathbf{P}_{1,2}=\left(M_{1,0}^{x}-a_{7} \cdot \text { FoB_Fwd_L } L, O^{y}-a_{8} \cdot \text { FoB_Trans, } O^{z}-T\right)^{T} \text {, } \\
& \mathbf{P}_{1,3}=\left(P_{1,2}^{x}, O^{y}-a_{9} \cdot \text { FoB_Trans, } P_{1,2}^{z}\right)^{T}, \\
& \mathbf{P}_{1,4}=\left(P_{1,3}^{x}, O^{y}-\frac{B}{2}, P_{1,3}^{z}\right)^{T} .
\end{aligned}
$$

290

where $\mathbf{O}=\left(O^{\mathrm{x}}, O^{\mathrm{y}}, O^{\mathrm{z}}\right)^{T}$ denotes the origin $(0,0,0)^{T}$ of the coordinate system, and $a_{\mathrm{i}}, i=0,1, . ., 9$, are internal parameters with $a_{1}<a_{7}, a_{2}<a_{3}$, and $a_{8}<a_{9}$. Regarding the functionality of these inequalities, they control the relative spatial positioning of control points, e.g., $a_{1}<a_{7}$, secures that $\mathbf{P}_{1,2}$ is placed towards $\mathrm{FP}$ and after $\mathbf{P}_{0,2}$; see Fig. 12. Otherwise, in case this inequality is violated, it is not unlikely that the control cage will suffer from self-intersections as a result of the fact that $\left\|\overrightarrow{\mathbf{M}_{\mathbf{1 , 2} 2} \mathbf{P}_{\mathbf{1 , 2}}}\right\|<\left\|\overrightarrow{\mathbf{M}_{\mathbf{1 , 2}} \mathbf{P}_{\mathbf{0}, 2}}\right\|$. In another example, if inequality $a_{2}<a_{3}$ is not satisfied, then the control polygon, depicted in red in Fig. 12, will be afar from the desired shape, which is that of a typical convex ship section. Furthermore, formulae (7)-(16) readily imply that mapping user-defined parameters to control points is done through linear mappings.

Once the control polygons of each of the transition curves $\mathcal{F} \mathcal{T} \mathcal{C}_{i}, i=0,1$, are defined, the connectivity between them and the vertices of the L-shaped control polygon $\mathcal{M}_{0}$ is set as shown in Fig. 12 . Despite the fact that all three control polygons do not share the same number of points, T-splines functionality 


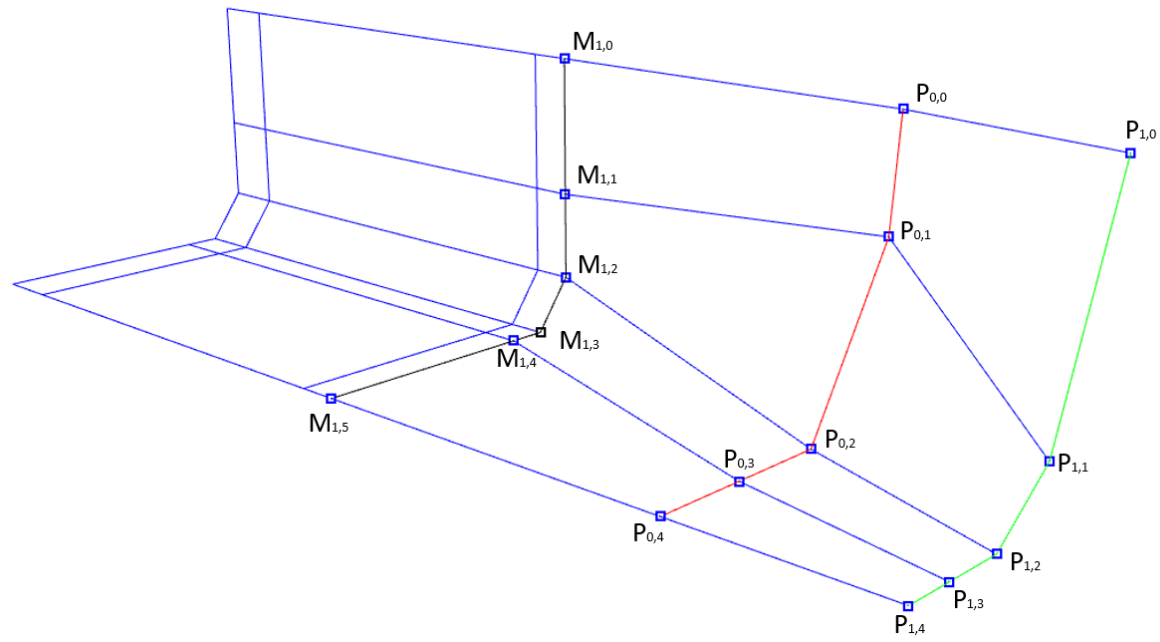

Figure 12: Control points of the L-shaped polygonal line $\mathcal{M}_{1}$ (depicted in black) and the first two Forward Transition Curves, $\mathcal{F} \mathcal{T} \mathcal{C}_{0}$ (depicted in red) and $\mathcal{F} \mathcal{T} \mathcal{C}_{1}$ (depicted in green).

enables us to circumvent this issue by introducing T-junctions, in this case at $\mathbf{M}_{1,3}$. Working in this way we are able to retain, in most cases, quadrilateral topology, i.e., create quadrilateral elements, with each of their control points having valence-4, to reduce the complexity and increase the quality of the developed surfaces.

In cases where a control point is necessary to be connected with fewer or more than 4 edges, extraordinary points have to be introduced. An example of such a control point of valence- 5 is shown in Fig. 13 . $\mathbf{b}_{0}$ is an extraordinary point on the Forward Perpendicular Transition Curve (depicted in magenta), connected with $\mathbf{b}_{1}$ of the first Bulb Curve (depicted in red).

The computed control cage is then fed to the T-splines plug-in, which in its turn delivers the corresponding ship hull instance. Figs. 14]16 depict a typical output with hollow rectangles indicating the control points $\mathbf{P}_{A}, A=0, \ldots, n$, of the obtained T-splines hull surface along with their connectivity (dashed lines), while solid lines constitute the mapping of T-mesh edges on the surface.

Considering the control points $\mathbf{P}_{A}, A=0, \ldots, n$, as the DoF of the surface 


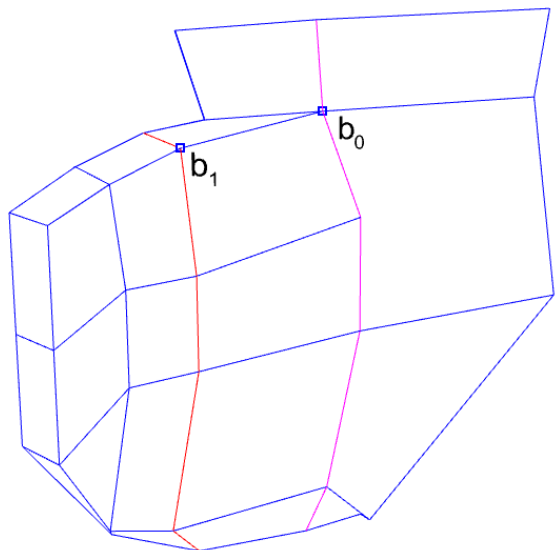

Figure 13: An example of a valence-5 extraordinary point. $\mathbf{b}_{0}$ is located on the Forward Perpendicular Transition Curve (depicted in magenta) and is connected with $\mathbf{b}_{1}$ of the first Bulb Curve (depicted in red).

representation (1), their population of which $(=n+1)$ is a measure of the computational complexity of the resulting surface. For the surface in Figs. 14 . 16. $n+1=196$. On the contrary, DoF of parent ship hull and CAESES's model 325 (the latter exported as an igs file from CAESES) is approximately 8,000 and 200,000 respectively. Since every T-splines can be accurately represented as a NURBS multi-patch surface, one can experience the DoF overloading effect of NURBS by transforming T-splines into NURBS and count the control points. For the surface in question this operation leads to a multi-patch NURBS surface with approximately 24,500 control points.

The quality of the outcome is assessed against a set of criteria of geometric (a-c) and ship-design (d-e) nature:

(a) GUI-aided checking of self-intersection failures;

(b) Smoothness: at least $G^{1}$-continuity;

(c) Fairness: smooth distribution of curvatures, proper sign of curvatures, e.g., nearly zero Gaussian curvature in the area of the cylindrical mid-part;

(d) Deviation from prescribed $k^{t h}$-order moments, e.g., volume $(k=0)$, centroid $(k=1)$, moment of inertia $(k=2)$; 
(e) Deviation from a prescribed SAC.

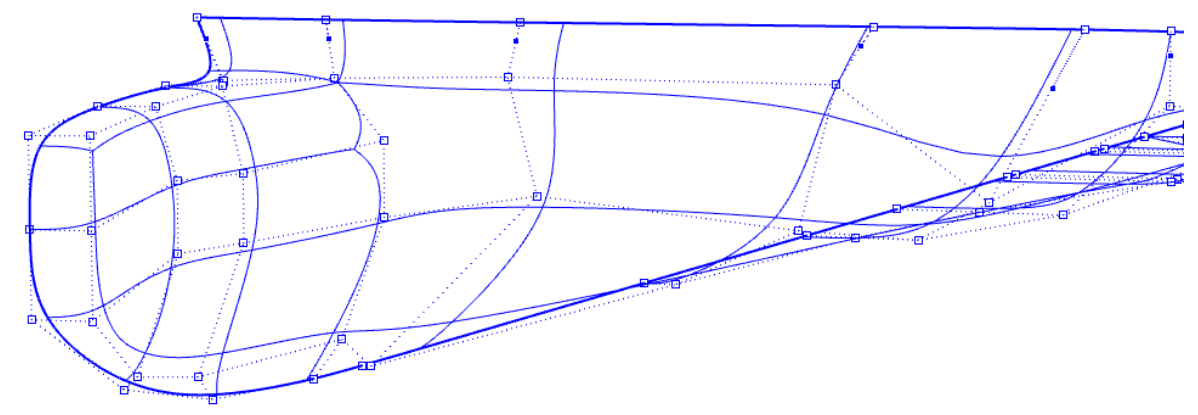

Figure 14: TshipPM output: perspective view from the bow with control points and on-surface mappings of T-mesh edges.

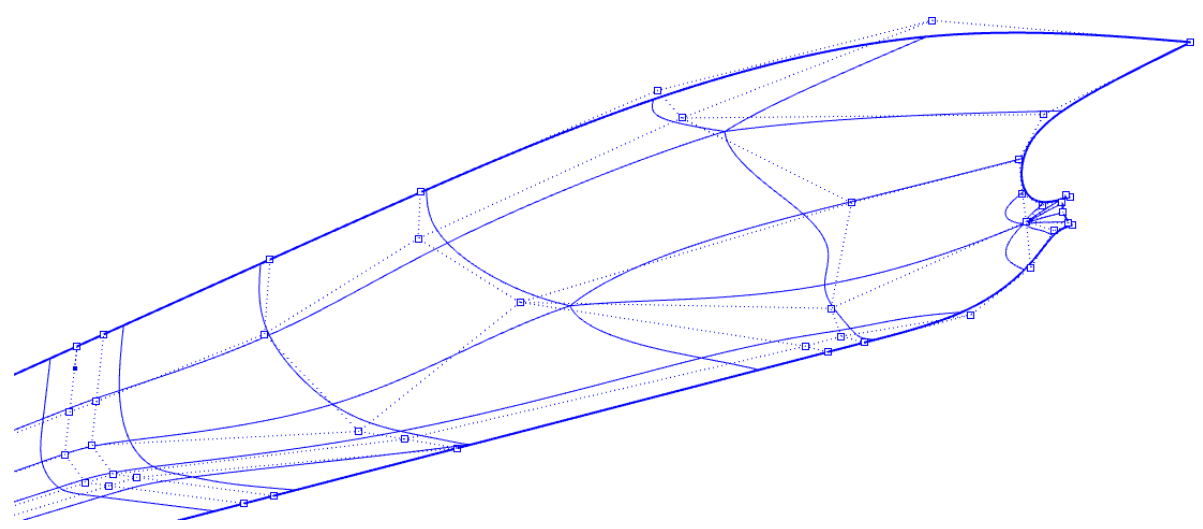

Figure 15: TshipPM output: perspective view from the stern.

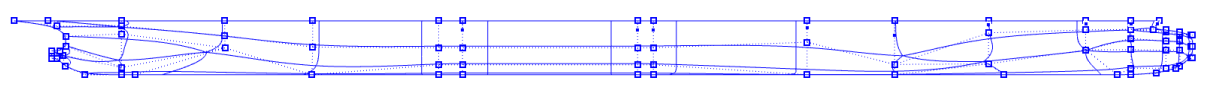

Figure 16: TshipPM output: side view.

\subsection{Behaviour of TshipPM against strong parameters' variation}

The question about which parameter values may lead to non-valid models, i.e., surfaces with self-intersections, is a "long standing" problem in parametric CAD design, which is easy to state but difficult to solve; see, e.g., [30. To provide an experimental indication of the robustness of the constructed PM, Figs. 17 
to 24 collect TshipPM's response to strong variations of 3 design parameters, namely the bilge radius (Bilge_R), the bulb length (Bulb_L) and the bulb height (Bulb_H). Variation is measured in percentage of the values corresponding to a parent hull, namely, the "well known" container-ship model KCS used in the literature for CAD and CFD benchmark purposes.

In particular, Fig. 17 and 18 depict TshipPM's response to Bilge_R decrease and increase by $30 \%$ respectively. Figs. 19,22 illustrate deviations of Bulb_H and Bulb_L up to $\pm 20 \%$. Finally, Fig. 23 shows a TshipPM instance where bulb_H has been decreased by $60 \%$, while Fig. 24 is an instance where both Bulb_H and Bulb_L have been decreased by $50 \%$.

\section{Comparison with CAESES}

This section focuses on comparing the performance of TshipPM and a well established commercial PM for ship design, namely CAESES [31]. This comparison will use the container-ship model KCS 27] as parent hull and the following criteria:

1. Common external parameters;

2. $k^{t h}$-order moments $(k=0,1,2)$, i.e., volume, volume centroid, moment of inertia;

3. SAC;

4. Gaussian curvature as a measure of surface shape and fairness;

5. Sectional curvature for assessing the shape and fairness of sections at specific longitudinal positions;

6. Hausdorff distance.

\subsection{CAESES parametric modeller}

CAESES adopts the classic naval architecture approach: a set of longitudinal lines - so-called basic curves - is laid out from which all information can be retrieved to subsequently establish the geometry of the ship hull. This is done in a 3-stage process: 


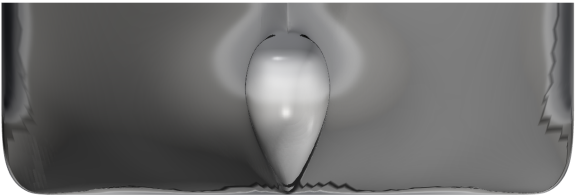

Figure 17: Decreasing $\widehat{\text { Bilge_}} R$ by $30 \%$.

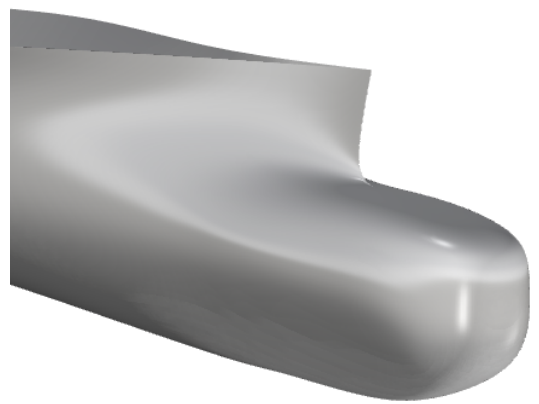

Figure 19: Decreasing $\widehat{B u l b \_H}$ by $20 \%$.

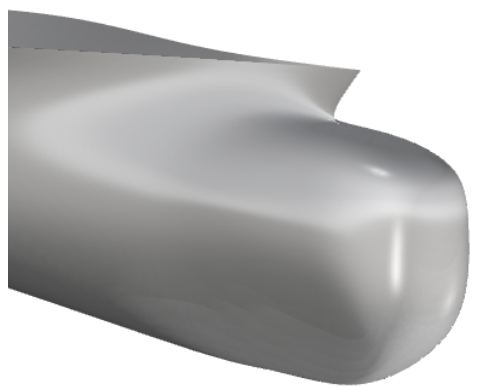

Figure 21: Decreasing $\widehat{\text { Bulb_L }}$ by $20 \%$.

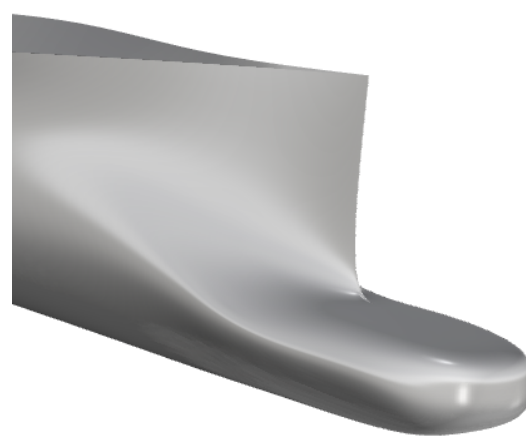

Figure 23: Decreasing $\widehat{B u l b \_H}$ by $60 \%$.

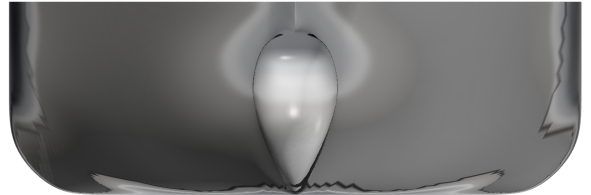

Figure 18: Increasing $\widehat{\text { Bilge_}} R$ by $30 \%$.

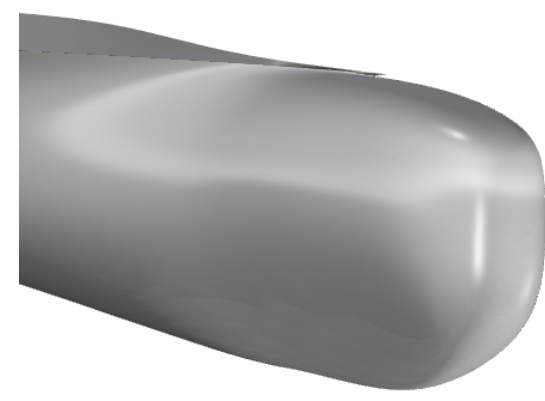

Figure 20: Increasing $\widehat{B u l b \_H}$ by $20 \%$.

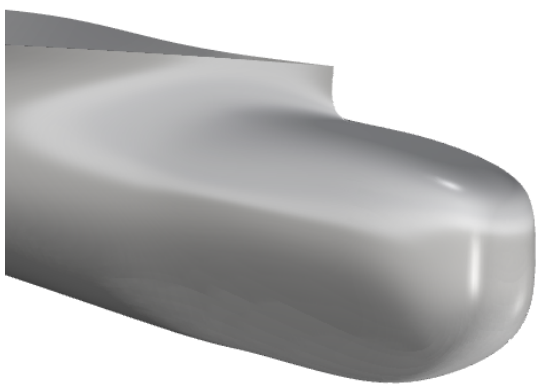

Figure 22: Increasing $\widehat{B u l b \_L}$ by $20 \%$.

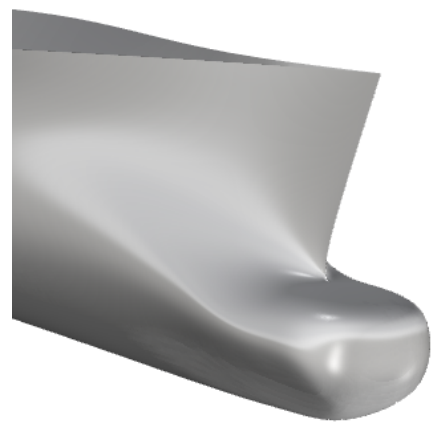

24

Figure 24: Decreasing both $\widehat{\text { Bulb_L} L}$ and $\widehat{B u l b \_} H$ by $50 \%$. 
1. Parametric design of a suitable set of basic curves such as deck profile, design waterline, flat of side (FoS) and flat of bottom (FoB), centerplane profile etc. The basic curves are built in agreement with a few prominent transversal curves, e.g., the main frame section, the transom, and, optionally, additional sections in the forward or afterward body.

2. Parametric modelling of design sections derived from the basic curves.

3. Generation of a set of B-spline surfaces that interpolate or closely approximate the design sections.

Curve design in CAESES is based on the concept of the $F$-spline curves 32. These curves are formulated considering a base of 4 parameters including the area, securing an inherently smooth and convex behaviour and reducing the need for further heuristic computations. Furthermore, Property Distribution Curves $(P D C)$ are introduced, representing the variation of important geometric properties of the hull form along, e.g., the longitudinal direction. Typical examples of PDC include SAC, the distribution curve of rise at the bottom, the design-waterline, deck profile and others.

In the present study, CAESES has been employed using 31 input (external in the terminology of TshipPM) parameters, depicted graphically in Fig. 25 and categorised in Table 2 in three groups, namely Main Dimensions, XmainFrame, Forebody and Aftbody parameters.
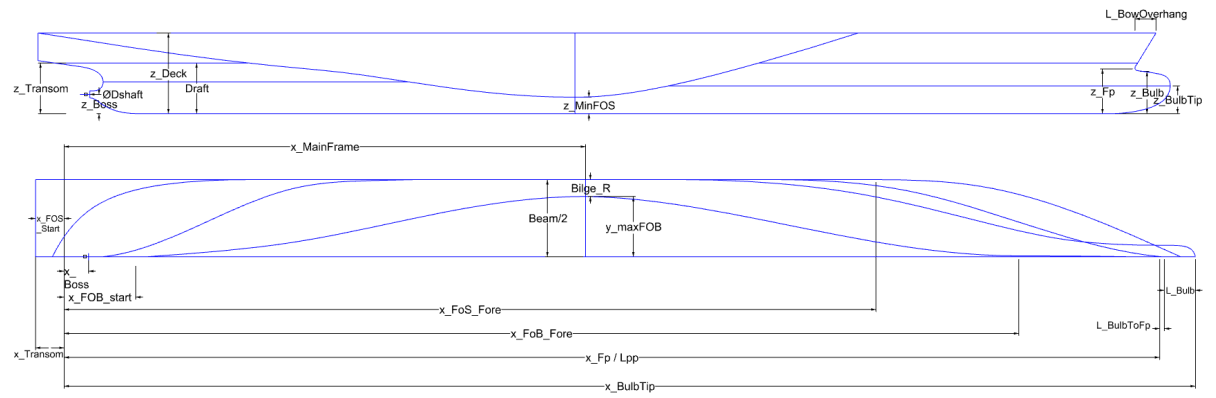

Figure 25: CAESES input parameters. 
Table 2: CAESES Parameters and Groups

\begin{tabular}{cc}
\hline Parameter & Description \\
\hline \hline Lpp & Main Dimensions \\
Beam & Distance between perpendiculars \\
Draft & Beam (breadth) of ship \\
z_Deck & Design waterline of the ship \\
y_MaxFOB & Deck height of the ship \\
y_MaxFOB_Fore_Factor & HalfBeam $\cdot$ y_MaxFOB_Fore_Factor \\
z_MinFOS & factor used in y_maxFOB \\
z_MinFOS_Fore_Factor & Height $\cdot$ zMinFOS_Fore_Factor \\
& factor used in z_MinFOS \\
x_MainFrame & X position of the largest section area \\
z_Bulb & x_FP $\cdot$ x_MainFrame_Factor \\
factor used in x_MainFrame
\end{tabular}


Table 2 - Continued from previous page

\begin{tabular}{cc}
\hline Name of Parameter & $\begin{array}{c}\text { Association to } \\
\text { Dimensional Parameters }\end{array}$ \\
\hline z_BulbTip & Bulb Tip Height \\
L_Bulb & Length of Bulbous Bow \\
L_BulbToFp & Distance from Bulbous Bow Tip to FP \\
L_BowOverhang & Length of Bow Overhang \\
\hline & Aftbody \\
\hline x_FOB_Start & x position of starting point of Flat of Bottom \\
x_FOB_Start_Factor & x_FP $\cdot$ x_FOB_Start_Factor \\
x_FOS_Start & factor used in x_FOB_Start \\
& x position of starting point of Flat of Side \\
x_FOS_Start_Factor & x_FP $\cdot$ x_FOS_Start_Factor \\
x_Boss & factor used in x_FOS_Start \\
x_Transom & x position of Shaft \\
z_Transom & x position of Transom \\
Dshaft & Transom Height \\
\end{tabular}

In the ensuing subsections $\S \S 4.2,4.6$ we present and discuss the results obtained from using TshipPM and CAESES for approximating the KCS parent hull. For ease of reference we call PM1 and PM2 the models produced by TshipPM and CAESES, respectively. All calculations have been conducted with models spatially located as shown in Fig. 26, with the tip of the bow overhang (most front part of the Lwl) being located at the start of the coordinate system.

\subsection{Common parameters}

Though the adopted geometric representation and the parametric-modelling methodology employed by TshipPM and CAESES are different, the two PMs 


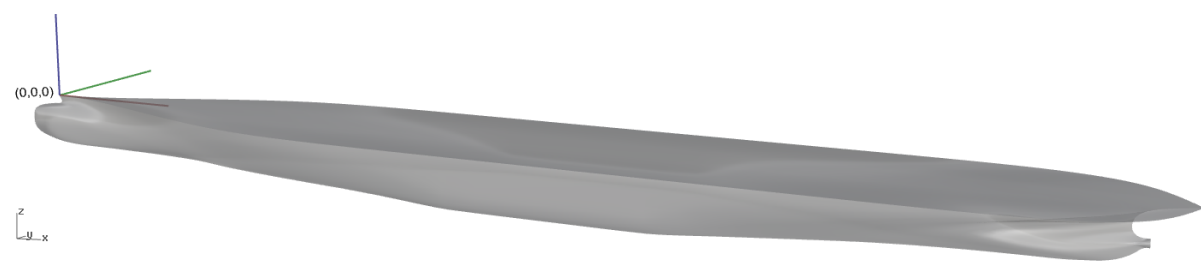

Figure 26: Coordinate system and models' orientation.

do share many common input parameters. In particular, the modellers have 15 common parameters ( $56 \%$ of TshipPM's and $48 \%$ of CAESES's parameters) 405 collected in Table 3 .

Table 3: TshipPM-CAESES common-parameter list

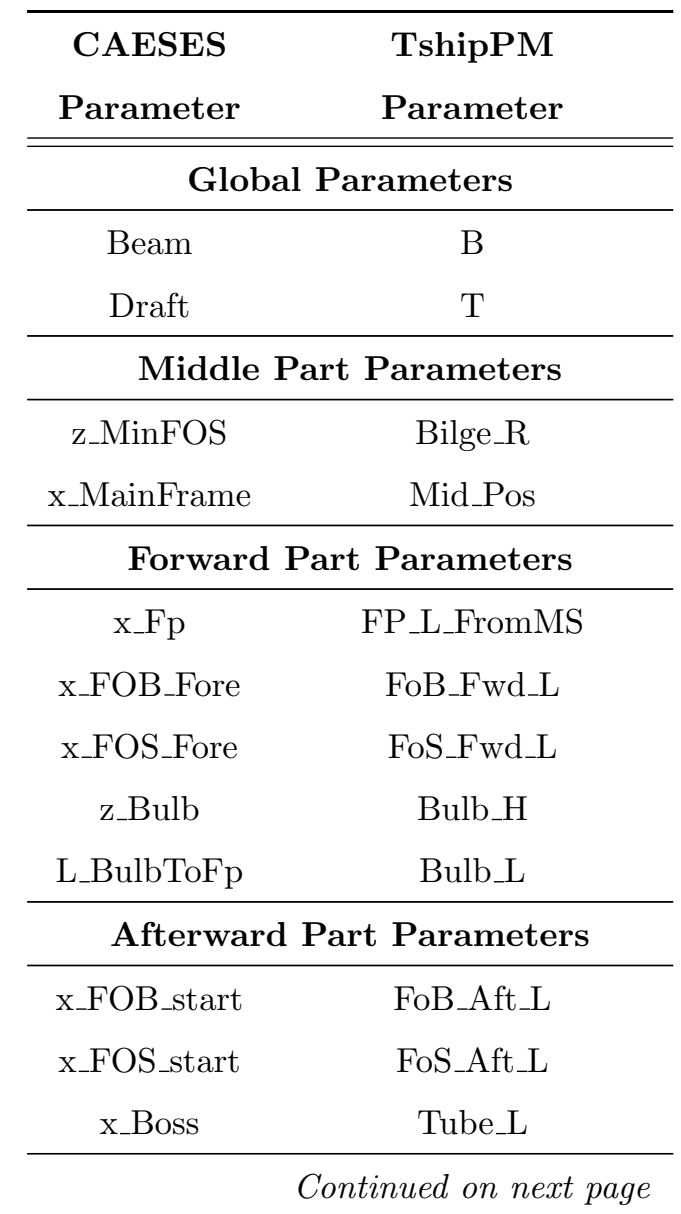


Table 3 - Continued from previous page

\begin{tabular}{cc}
\hline x_Transom & Stern_L \\
z_Transom & Transom_H \\
Dshaft & Tube_D \\
\hline
\end{tabular}

\subsection{Moments comparison}

In this subsection we present the results from comparing volumetric moments up to $2^{\text {nd }}$ order, provided by PM1 and PM2 against the KCS parent hull. inertia, PM1's estimate differs from that of the parent hull by $\delta I_{x}=0.180 \%$, $\delta I_{y}=-0.514 \%$ and $\delta I_{z}=-0.496 \%$. For PM2, the corresponding quantities vary by $1.288 \%, 0.756 \%, 0.767 \%$.

For the segmented hull, PM1 shows satisfactory behaviour for all calculated 430

Comparison has been conducted both for the whole ship hulls and for their segmented parts, namely forward $(x \in[-6.7 m, 102.8 m])$, middle part $(x \in$ $[102.8 m, 146.3 m])$ and afterward part $(x \in[146.3 m$, total length $])$. PM1 and parent ship hull moments have been calculated via Rhino3D, while PM2 moments have been calculated using ANSYS Fluent ${ }^{\circledR}$. Negative differences indicate that the parent ship hull value is smaller than the corresponding value of PM1 or PM2 and vice versa. The obtained values and resulting deviations are collected in Tables 4 and 5 .

Focusing on the whole ship hull, volume, volume centroid, and moment of inertia for both PM1 and PM2 lie very close to those of the parent hull, with differences less than $1 \%$. For volume, PM1 overestimates about $0.119 \%$, while PM2's estimate differs by $0.073 \%$. As far as centroid location is concerned, PM1's estimate deviates by $0.063 \%$ and $-0.090 \%$ along the $x-$ and $z$-axis respectively, while PM2's centroid estimate exhibits a variation of $0.038 \%$ and $-0.001 \%$, correspondingly. For both parametric models and the parent hull, the $y$-coordinate of centroid is zero in single precision accuracy. In terms of moments and for all three segments as differences of less than $1 \%$ occur for 
all calculated moments. Variations of more than $1 \%$ occur for the middle and afterward parts of PM2, as far as $2^{\text {nd }}$ order moments are concerned. Especially at the middle part $\delta I_{x}=2.449 \%, \delta I_{y}=2.194 \%, \delta I_{z}=2.007 \%$, while for afterward part corresponding deviations are $1.675 \%, 1.644 \%$, and $1.649 \%$.

Table 4: Comparison of moments among parent ship-hull, PM1 and PM2 models (full model).

\begin{tabular}{|c|c|c|c|c|c|}
\hline Models & $\begin{array}{l}\text { Volume } \\
\qquad\left(\mathrm{m}^{3}\right)\end{array}$ & & $\begin{array}{c}\text { Volume } \\
\text { Centroid } \\
(\mathrm{m})\end{array}$ & & $\begin{array}{c}\text { Inertia } \\
\left(\mathrm{m}^{5}\right)\end{array}$ \\
\hline \multirow{3}{*}{ Parent Ship } & \multirow{3}{*}{52,053} & $\mathrm{x}:$ & 118.407 & Ix: & $3,892,800$ \\
\hline & & $\mathrm{y}:$ & 0 & Iy: & $130,372,480$ \\
\hline & & $\mathrm{z}:$ & -4.894 & Iz: & $133,304,065$ \\
\hline \multirow{3}{*}{ PM1 } & \multirow{3}{*}{52,115} & $\mathrm{x}:$ & 118.332 & Ix: & $3,885,819$ \\
\hline & & $\mathrm{y}:$ & 0 & Iy: & $131,046,043$ \\
\hline & & $\mathrm{z}:$ & -4.899 & Iz: & $133,967,881$ \\
\hline \multirow{3}{*}{ PM2 } & \multirow{3}{*}{52,015} & $\mathrm{x}:$ & 118.290 & Ix: & $3,843,304$ \\
\hline & & $\mathrm{y}:$ & 0 & Iy: & $129,394,600$ \\
\hline & & $\mathrm{z}:$ & -4.896 & Iz: & $132,289,720$ \\
\hline Difference (\%) & \multirow{3}{*}{-0.119} & $\mathrm{x}:$ & 0.063 & Ix: & 0.180 \\
\hline PM1 vs & & $\mathrm{y}:$ & 0 & Iy: & -0.514 \\
\hline Parent Ship & & $\mathrm{z}:$ & -0.090 & Iz: & -0.496 \\
\hline Difference (\%) & \multirow{3}{*}{0.073} & $\mathrm{x}:$ & 0.038 & Ix: & 1.288 \\
\hline PM2 vs & & $\mathrm{y}:$ & 0 & Iy: & 0.756 \\
\hline Parent Ship & & $\mathrm{z}:$ & -0.001 & Iz: & 0.767 \\
\hline
\end{tabular}

Notes: Inertia has been calculated with respect to Centroid Coordinate Axis. Negative percentage indicates that parent ship-hull's value is lower than that of PM1/2, vice versa for positive values. 
Table 5: Comparison of moments among parent ship-hull, PM1 and PM2 models. (Segmentation at $\mathrm{x}^{1}=102.8 \mathrm{~m}$, and $\mathrm{x}^{2}=146.3 \mathrm{~m}$ )

\begin{tabular}{lccc}
\hline & Volume & Volume & Inertia \\
Models & $\left(\mathrm{m}^{3}\right)$ & Centroid & $\left(\mathrm{m}^{5}\right)$ \\
& & $(\mathrm{m})$ & \\
\hline
\end{tabular}

\begin{tabular}{|c|c|c|c|c|c|}
\hline \multicolumn{6}{|c|}{ Forward Part } \\
\hline & & $\mathrm{x}$ : & 66.570 & Ix: & $1,281,763$ \\
\hline \multirow[t]{3}{*}{ Parent Ship } & 20,139 & $y:$ & 0 & Iy: & $12,289,833$ \\
\hline & & $\mathrm{z}:$ & -4.902 & Iz: & $13,213,492$ \\
\hline & & $\mathrm{x}:$ & 66.365 & Ix: & $1,273,039$ \\
\hline \multirow[t]{3}{*}{ PM1 } & 20,167 & $y:$ & 0 & Iy: & $12,306,184$ \\
\hline & & $\mathrm{z}:$ & -4.900 & Iz: & $13,220,480$ \\
\hline & & $\mathrm{x}:$ & 66.554 & Ix: & $1,273,460$ \\
\hline \multirow[t]{2}{*}{ PM2 } & 20,100 & $y:$ & 0 & Iy: & $12,266,331$ \\
\hline & & $\mathrm{z}:$ & -4.896 & Iz: & $13,183,772$ \\
\hline Difference (\%) & & $\mathrm{x}:$ & 0.309 & Ix: & 0.685 \\
\hline PM1 vs & -0.136 & $\mathrm{y}:$ & 0 & Iy: & -0.133 \\
\hline Parent Ship & & $\mathrm{z}:$ & 0.037 & Iz: & -0.053 \\
\hline Difference (\%) & & $\mathrm{x}$ : & 0.025 & Ix: & 0.652 \\
\hline PM2 vs & 0.198 & $\mathrm{y}:$ & 0 & Iy: & 0.192 \\
\hline Parent Ship & & $\mathrm{z}:$ & 0.131 & Iz: & 0.225 \\
\hline
\end{tabular}

\begin{tabular}{|c|c|c|c|c|c|}
\hline \multicolumn{6}{|c|}{ Middle Part } \\
\hline \multirow{3}{*}{ Parent Ship } & \multirow{3}{*}{14,993} & $\mathrm{x}:$ & 123.972 & Ix: & $1,393,339$ \\
\hline & & $\mathrm{y}:$ & 0 & Iy: & $2,550,009$ \\
\hline & & $\mathrm{Z}:$ & -5.308 & Iz: & $3,658,949$ \\
\hline \multirow{4}{*}{ PM1 } & \multirow{4}{*}{15,034} & $\mathrm{x}:$ & 123.998 & Ix: & $1,402,369$ \\
\hline & & $\mathrm{y}:$ & 0 & Iy: & $2,565,262$ \\
\hline & & $\mathrm{Z}:$ & -5.322 & Iz: & $3,681,224$ \\
\hline & & $\mathrm{x}$ : & 123.974 & Ix: & $1,360,028$ \\
\hline
\end{tabular}


Table 5 -Continued from previous page

\begin{tabular}{|c|c|c|c|c|c|}
\hline Models & $\begin{array}{l}\text { Volume } \\
\qquad\left(\mathrm{m}^{3}\right)\end{array}$ & & $\begin{array}{c}\text { Volume } \\
\text { Centroid } \\
(\mathrm{m})\end{array}$ & & $\begin{array}{c}\text { Inertia } \\
\left(\mathrm{m}^{5}\right)\end{array}$ \\
\hline \multirow[t]{2}{*}{ PM2 } & 14,963 & $\mathrm{y}:$ & 0 & Iy: & $2,495,260$ \\
\hline & & $\mathrm{z}:$ & -5.302 & Iz: & $3,586,954$ \\
\hline Difference (\%) & & $\mathrm{x}:$ & 0.021 & Ix: & -0.644 \\
\hline PM1 vs & -0.273 & $\mathrm{y}:$ & 0 & Iy: & -0.595 \\
\hline Parent Ship & & $\mathrm{z}:$ & -0.261 & Iz: & -0.605 \\
\hline Difference (\%) & & $\mathrm{x}:$ & -0.002 & Ix: & 2.449 \\
\hline PM2 vs & 0.198 & $\mathrm{y}:$ & 0 & Iy: & 2.194 \\
\hline \multirow[t]{3}{*}{ Parent Ship } & & $\mathrm{z}:$ & 0.102 & Iz: & 2.007 \\
\hline & & wa & Part & & \\
\hline & & $\mathrm{x}:$ & 175.178 & Ix: & $1,212,449$ \\
\hline \multirow[t]{3}{*}{ Parent Ship } & 16,919 & $\mathrm{y}:$ & 0 & Iy: & $6,415,948$ \\
\hline & & $\mathrm{z}:$ & -4.519 & Iz: & $7,319,577$ \\
\hline & & $\mathrm{x}:$ & 175.260 & Ix: & $1,205,310$ \\
\hline \multirow[t]{3}{*}{ PM1 } & 16,913 & $\mathrm{y}:$ & 0 & Iy: & $6,411,417$ \\
\hline & & $\mathrm{z}:$ & -4.521 & Iz: & $7,308,097$ \\
\hline & & $\mathrm{x}:$ & 175.162 & Ix: & $1,192,473$ \\
\hline \multirow[t]{2}{*}{ PM2 } & 16,951 & $\mathrm{y}:$ & 0 & Iy: & $6,312,204$ \\
\hline & & $\mathrm{z}:$ & -4.512 & Iz: & $7,200,846$ \\
\hline Difference (\%) & & $\mathrm{x}:$ & -0.047 & Ix: & 0.592 \\
\hline PM1 vs & 0.037 & $\mathrm{y}:$ & 0 & Iy: & 0.071 \\
\hline Parent Ship & & $\mathrm{z}:$ & -0.049 & Iz: & 0.157 \\
\hline Difference (\%) & & $\mathrm{x}:$ & 0.009 & Ix: & 1.675 \\
\hline PM2 vs & -0.187 & $\mathrm{y}:$ & 0 & Iy: & 1.644 \\
\hline Parent Ship & & $\mathrm{z}:$ & 0.161 & Iz: & 1.649 \\
\hline
\end{tabular}


positive values.

\subsection{SAC comparison}

Plotting and comparing SAC among different ship hulls is a useful tool for evaluating the longitudinal distribution of sectional area values (SAV) as well as extracting the longitudinal positions where the major variations of SAVs exist. Fig. 27 illustrates SAC of PM1 (red curve), PM2 (blue curve) and parent (green curve) ship hulls. It also shows the difference of SAV between PM1 and parent hull (magenta curve), as well as between PM2 and parent (black curve) with the maximum deviation $\left|S A V_{P M 1}-S A V_{\text {parent }}\right|_{\max }=6.51 \mathrm{~m}^{2}$ occurring at $x=50 \mathrm{~m}$, and $\left|S A V_{P M 2}-S A V_{\text {parent }}\right|_{\max }=3 \mathrm{~m}^{2}$ occurring at $x=50 \mathrm{~m}$ which corresponds to $1.9 \%$ and $8 \%$ of the maximum SAV respectively. It is also worth noticing that the red curve is constant along the interval $102.8 \leq x \leq 146.3$ as a result of the fact that TshipPM imposes by construction a strictly cylindrical part along the middle part of the hull and, as a result, possesses constant SAV in this interval.

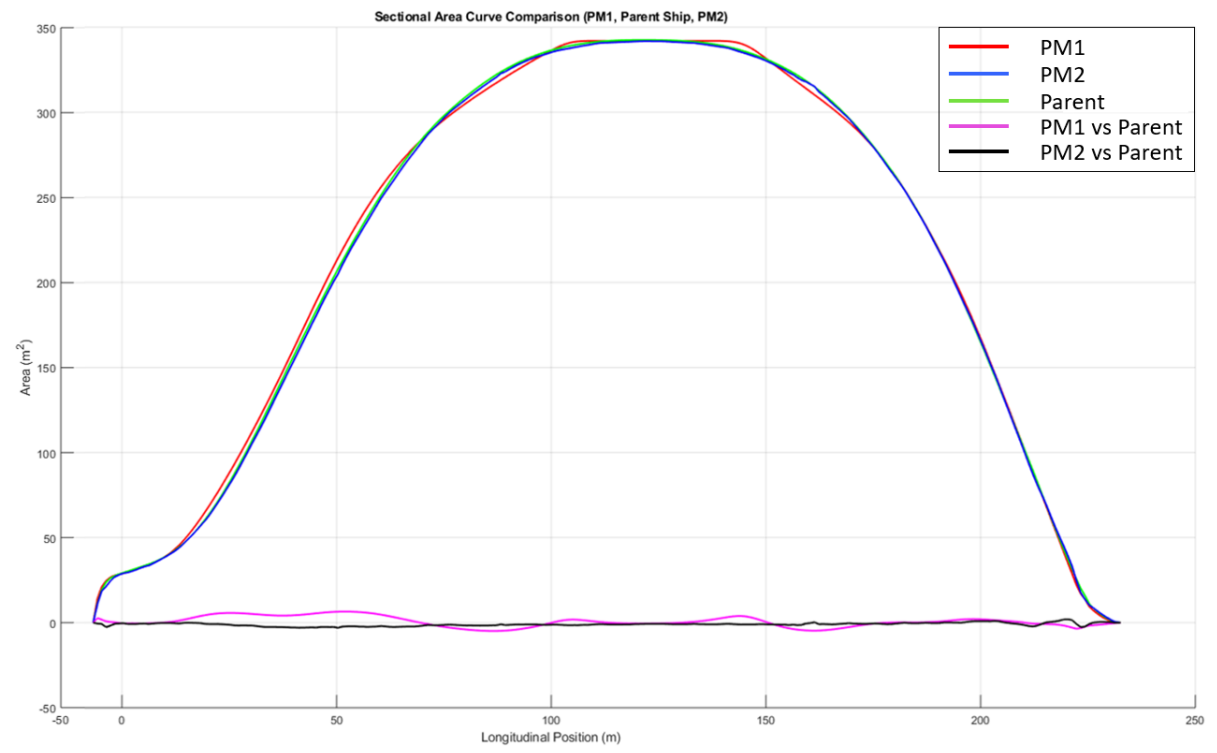

Figure 27: SAC comparison among PM1 (red curve), PM2 (blue curve) and the parent hull (green curve): the magenta curve depicts the deviation $S A V_{P M 1}-S A V_{P M 2}$ with the absolute maximum deviation occurring at $x=50 \mathrm{~m}$ (dashed line). 


\subsection{Gaussian-curvature comparison}

Gaussian curvature is an intrinsic geometrical property of surfaces used by designers to evaluate the fairness of the boundary of a 3D object and locate deficiencies versus the anticipated shape of the surface under consideration. The Gaussian curvature $K(\mathbf{P})$ at a point $\mathbf{P}$ of a smooth surface can be expressed as:

$$
\kappa(\mathbf{P})=\kappa_{1}(\mathbf{P}) \cdot \kappa_{2}(\mathbf{P})
$$

where $\kappa_{i}, i=1,2$ are the principal curvatures at $\mathbf{P}$.

In order to use a color map capable to easily reveal sign variations of the Gaussian curvature, a short interval around $\kappa=0$ has been selected, i.e., $\left[-10^{-6}, 10^{-6}\right]$. Blue color illustrates areas of negative $\kappa$, i.e., principal curvatures are of opposite sign, implying that the surface has locally a saddle-like (non-convex) shape. On the contrary, red color implies that both principal curvatures share the same sign, equivalently the surface is locally convex resembling the shape of a sphere. Finally, green color is used when $\kappa=0$, in which case the surface is cylindrical or flat. The color map range is illustrated in Fig. 31 and it is the same for Figs $28 \mid 33$,

Figs. 28 31 depict several (side, bow, stern and bottom) views of the Gaussian curvature plot of the surfaces of PM1 and PM2. In general, one can easily spot extraneous Gaussian-curvature sign variations in several areas of both plots. A more focused view, though, reveals that the Gaussian-curvature distribution of PM2 exhibits more extraneous small-scale oscillations between red (convex) and blue (non-convex) areas; see; Figs. 32,33 This may be attributed to the fact that CAESES constructs multi-patch NURBS surfaces of high complexity, consisting of many tiny patches, resulting to unevenly spaced isoparametric curves, and thus it is more challenging to achieve fairness in transition regions. For example, in Fig. 32 one can observe curvature oscillations at the midand bottom-stern which consist of a total of 50 patches. Nevertheless, Fig. 31 indicates that, in large scale, the shape of the flat of side/bottom provided by PM2 is better than that of PM1. In this connection it should be noted that 
${ }_{480}$ TshipPM imposes by construction a strictly cylindrical parallel middle part, indicated by the green rectangle in Fig. 31 .

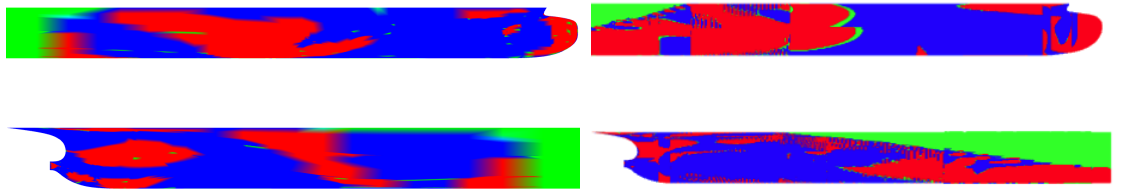

Figure 28: Side view of Gaussian-curvature plot of Fwd_Part (up) and Aft_Part (bottom) of PM1 (left) and PM2 (right).

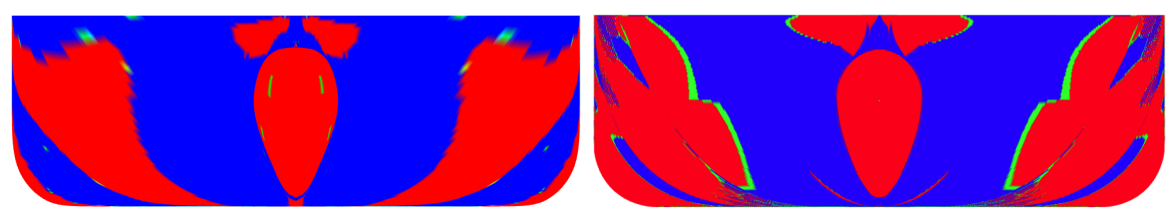

Figure 29: Bow view of Gaussian-curvature plot of PM1 (left) and PM2 (right).
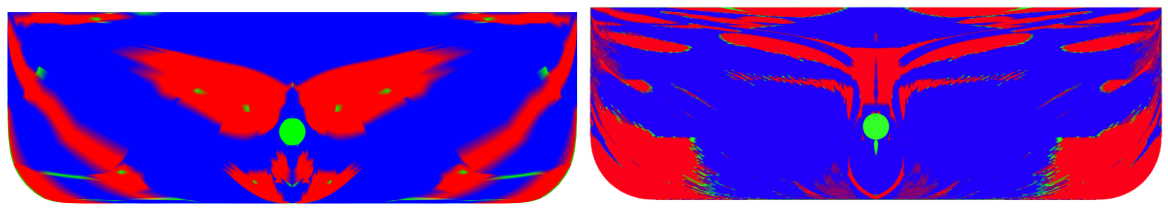

Figure 30: Stern view of Gaussian-curvature plot of PM1 (left) and PM2 (right).

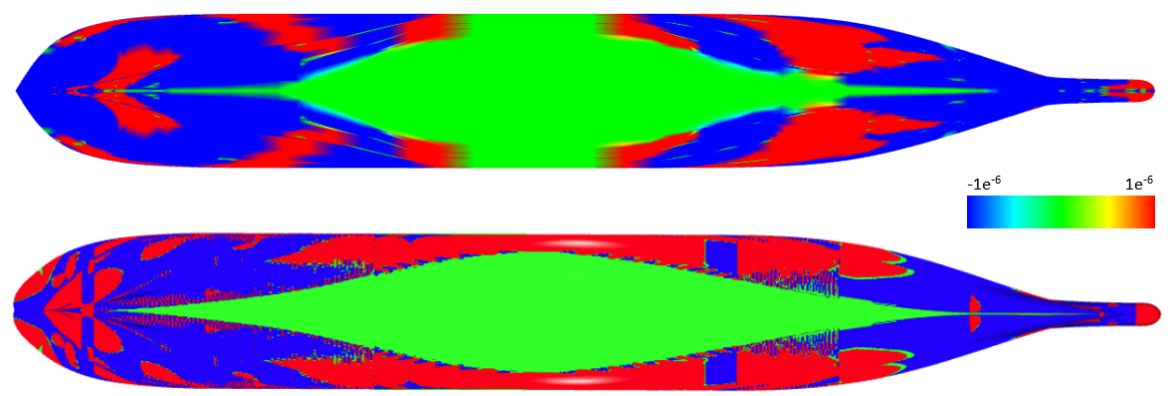

Figure 31: Bottom view of Gaussian-curvature plot of PM1 (top) and PM2 (bottom). Color map is the same for Figs 2833 


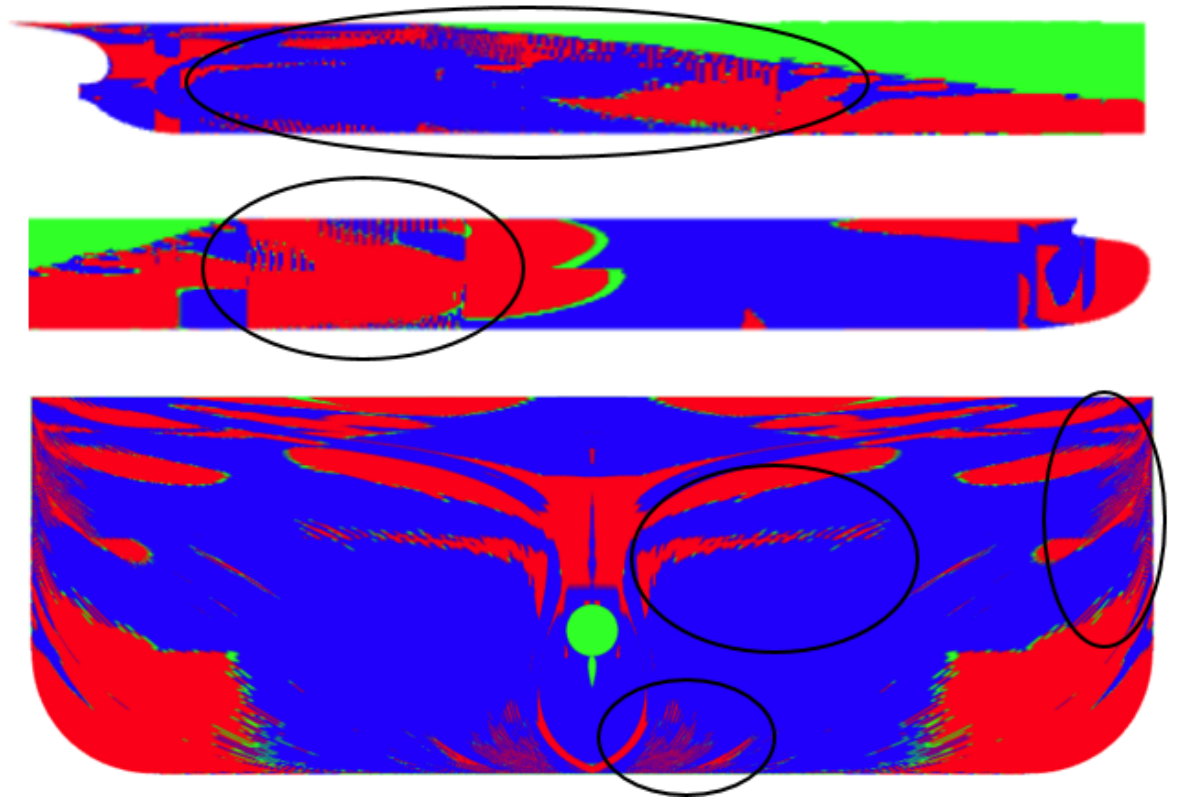

Figure 32: Small-scale extraneous oscillations of the Gaussian-curvature distribution of PM2: side (top) and stern view (bottom).

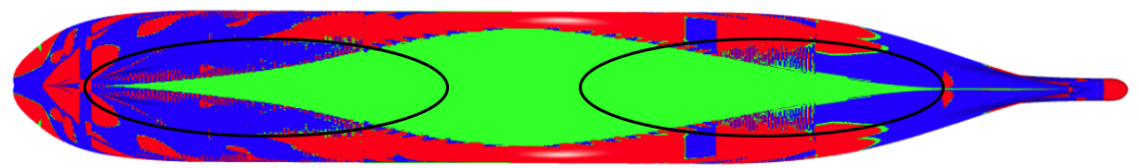

Figure 33: Small-scale extraneous oscillations of the Gaussian-curvature distribution of PM2: bottom view.

\subsection{Sectional-curvature comparison}

By sectional curvature we refer to the curvature distribution of the planar curves obtained by intersecting the ship hull with planes vertical to its longitudinal axis. When selected at prescribed distances, called stations, the resulting family of curves is referred to as the body plan of the hull. In design practice, the fairness of these sections is evaluated via their curvature distribution depicted as a function of a parameter running along the curve or in the porcupine format, adopted herein. Figs. 34 37 indicate that PM1 and PM2 provide, in general, 
fair sections which are also free from extraneous inflection points, with exception to PM2's sections at $x=146.3 m$ and $x=205.3 m$, as shown in Fig. 38 .
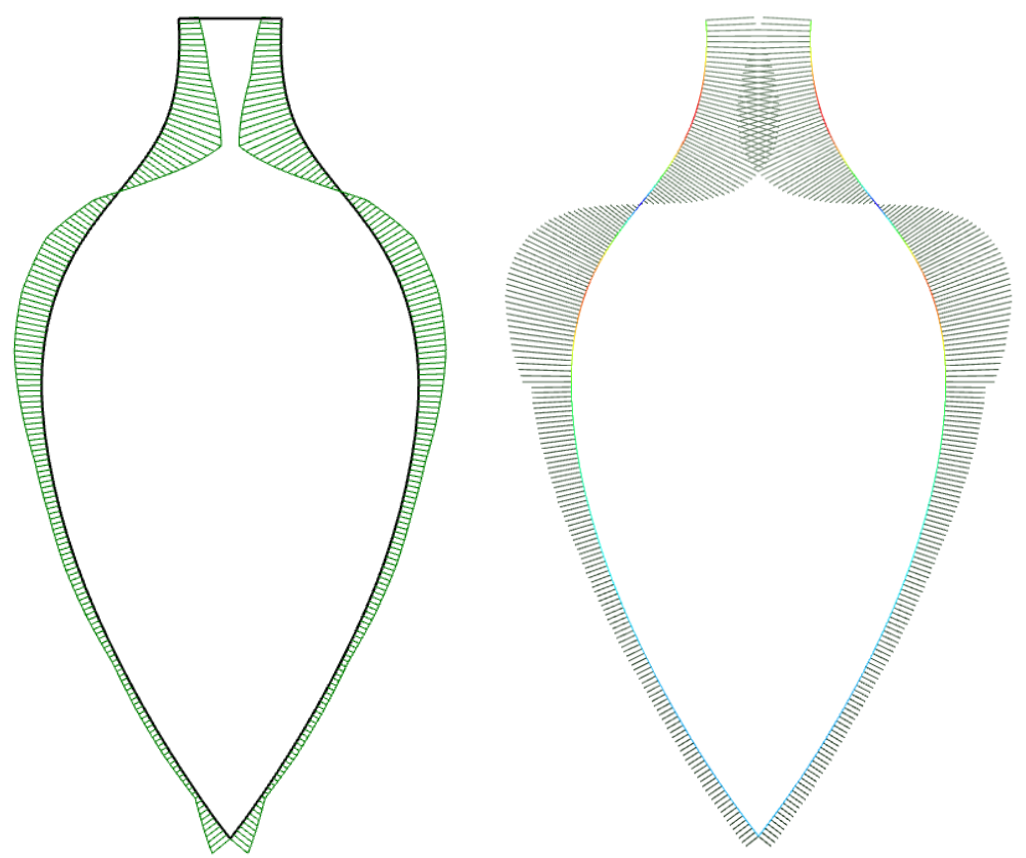

Figure 34: PM1 (left), and PM2 (right) porcupine plots of section at $x=-1 \mathrm{~m}$.

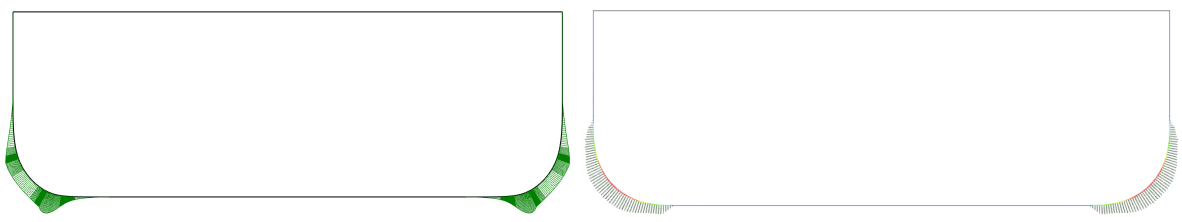

Figure 35: PM1 (left), and PM2 (right) porcupine plots of section at $x=102.8 \mathrm{~m}$.

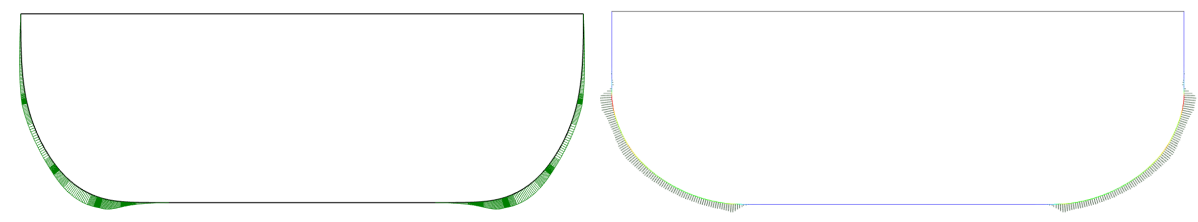

Figure 36: PM1 (left), and PM2 (right) porcupine plots of section at $x=146.3 \mathrm{~m}$. 

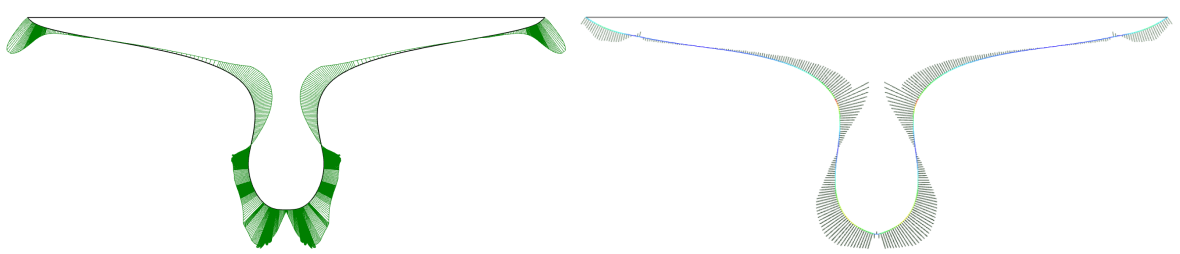

Figure 37: PM1 (left), and PM2 (right) porcupine plots of section at $x=205.3 \mathrm{~m}$.

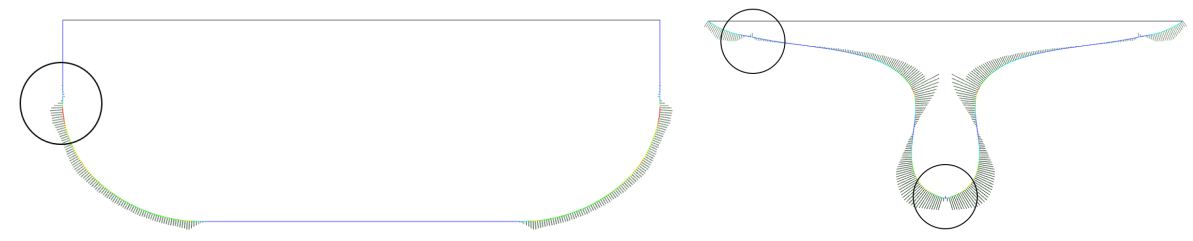

Figure 38: Extraneous inflection points of PM2 for sections at $x=146.3 m$ (left) and $x=$ $205.3 m$ (right).

\subsection{Hausdorff distance comparison}

In this subsection we adopt the concept of Hausdorff distance for comparing PM1 and PM2 in terms of their geometric deviation from the parent hull (KCS). Using the MeshLab ${ }^{\circledR}$ tool 9 and a dense sampling on the surfaces involved $\left(3.6 \times 10^{5}\right.$ points $)$, Fig. 39 depicts the distribution of deviation $\delta(\mathbf{x})$ of PM1 and PM2 from the parent hull, defined as:

$$
\delta_{i}(\mathbf{x})=\min _{\mathbf{y} \in \mathbf{P K C S}} \mathbf{d}(\mathbf{x}, \mathbf{y}), \quad \mathbf{x} \in \mathrm{PM}_{\mathbf{i}}, \mathrm{i}=1,2,
$$

where $d(\mathbf{x}, \mathbf{y})$ denotes the Euclidean distance between two points $\mathbf{x}$ and $\mathbf{y}$. The color map ranges over $[0,0.6]$, which covers the maximum deviation measured for PM1 (top pair in Fig 39. The mean deviation is $0.078 \mathrm{~m}$ for PM1 and $0.016 \mathrm{~m}$ for PM2 (bottom pair in Fig 39 ). Finally, the Hausdorff distance between PM1/PM2 and the parent hull is equal to $0.6 \mathrm{~m} / 0.24 \mathrm{~m}$ respectively.

The obtained results indicate a considerably better behaviour for PM2, attributed to the large DOF (196 for PM1 vs $O\left(10^{5}\right)$ for PM2. PM1's significant

\footnotetext{
${ }^{9}$ http://www.meshlab.net/
} 
deviations occur in two areas, namely the transition area from mid-ship towards the afterward part of the hull, and the stern area. Large deviations in the first area are attributed to the fact that PM1 surface changes abruptly from the cylindrical middle body towards, especially, the afterward part; see also Fig. 31 (top). As for the deviation in the stern area, PM2 performs better but at the expense of fairness; see Fig. 31 (bottom).

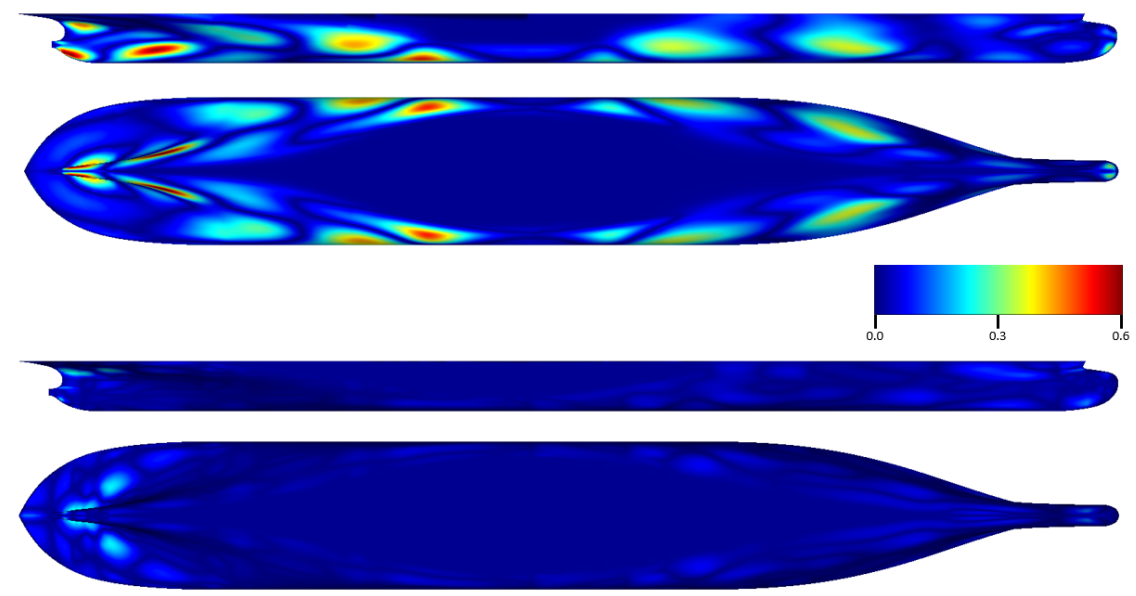

Figure 39: Distance plot of PM1 (top pair) and PM2 (bottom pair) with respect to the parent ship hull.

\section{Discussion and Future Work}

In this paper a T-splines parametric modeller (PM) for ship hulls is presented analytically and tested versus a commercial PM (CAESES). TshipPM is an improved version of that in [1], enabling more flexibility for handling challenging areas of the ship hull geometry, such as the bow, stern, and transition areas from mid-ship towards forwards and afterward perpendiculars.

TshipPM uses 27 parameters accessible to the user. Besides the basic dimensions, Lwl, B, and T, the remaining parameters are non-dimensional, valued in $(0,1)$. All parameters are appropriately interrelated so that a consistent control 515 cage is delivered to Rhino5 3D and T-splines plug-in, which produce surfaces consisting of only 196 control points. As a result, TshipPM is able to navigate 
in a rich design space at a low computational cost. Extensive experimentation also indicates that TshipPM is stable for it provides valid outcomes (non-selfintersecting geometries) against strong parameter variations. TshipPM is compared against CAESES which uses 31 parameters for the representation of the container-ship hull KCS [27], already employed in the pertinent literature for CAD and CFD benchmarking purposes. Comparison criteria include: a) common parameters, b) moments (up to $2^{\text {nd }}$ order), c) sectional area curve (SAC), d) Gaussian and e) sectional curvatures, and f) Hausdorff distance. Both PMs behave satisfactory, with TshipPM doing better with respect to moments, producing $\mathrm{G}^{1}$-continuous surfaces, which exhibit less undesired sign variations of the Gaussian and sectional curvatures. Furthermore, TshipPM produces instances with considerably fewer control points in comparison with CAESES, as it takes advantage of the T-splines representation. CAESES, on 530 the other hand, performs better with respect to the deviation from the KCS SAC, as well as the fairness of sections, except from some extraneous inflection points. Finally, CAESES produces accurate planar areas for flat of side/bottom, though at the expense of smoothness, and its geometric deviation from KCS, measured in terms of Hausdorff distance, is considerably smaller. Next steps for the further development of the TshipPM include:

(a) Automatic determination of parameter values so that a given parent hull can be re-constructed via minimising the deviation from moments, SAC, and appropriate geometric distance;

(b) Derivation of sufficient conditions of the control cage which secure that the corresponding T-splines surface does not suffer form self-intersections;

(c) Decrease of extra-ordinary vertices under complexity constraints;

(d) Accurate incorporation of planar areas.

Acknowledgements: The first and last author of this work has received funding from the European Union's Horizon 2020 research and innovation pro${ }_{545}$ gramme under the Marie Skłodowska-Curie grant ARCADES (agreement No $675789)$. 


\section{References}

[1] K. Kostas, A. Ginnis, C. Politis, P. Kaklis, Ship-hull shape optimization with a T-spline based BEM-isogeometric solver, Computer Methods in Applied Mechanics and Engineering 284 (2015) 611-622. doi: $10.1016 / \mathrm{j} . \mathrm{cma} .2014 .10 .030$.

[2] H. Nowacki, Five decades of computer-aided ship design, Computer-Aided Design 42 (2010) 956-969. doi:10.1016/j.cad.2009.07.006.

[3] H. Nowacki, C. Creutz, F. Munchmeyer, Lines creation by computer - objectives, methods and results, in: Proceedings of the Ship-Symposium on Computer-Aided Hull Surface Definition, Annapolis, MD, USA, 1977.

[4] H. Lackenby, On the systematic geometrical variation of ship form, in: Transactions of the Royal Institute of Naval Architects (RINA), Vol. 92, 1950. doi:10.5574/KSOE.2013.27.2.001.

[5] S. Harries, H. Nowacki, Form-parameter approach to the design of fair hull shapes, Cambridge, MA, USA, 1999.

[6] H.-C. Kim, Parametric design of ship hull forms with a complex multiple domain surface topology, Ph.D. thesis, Berlin, Germany (2004).

[7] C. Abt, S. Harries, A new approach to integration of CAD and CFD for naval architects, in: 6th International Conference on Computer Applications and Information Technology in Maritime Industries (COMPIT), Cortona, Italy, 2007.

[8] S. Harries, Serious play in ship design - tradition and future of ship design, in: Berlin Colloquium, Berlin, Germany, 2008.

[9] P. Zhang, D.-X. Zhu, Parametric approach to design of hull forms, Journal of Hydrodynamics 20 (2008) 804-810. doi:10.1016/S1001-6058(09) 60019-6 
[10] H. Koelman, I. Horváth, A. Aalbers, Hybrid representation of the shape of ship hulls, International Shipbuilding Progress 48 (2001) 247-269.

[11] D. Forsey, R. Bartels, Hierarchical B-spline refinement, Computer Graphics 22 (1988) 205-212. doi:10.1145/54852.378512.

[12] J. Deng, F. Chen, X. Li, C. Hu, W. Tong, Z. Yang, Y. Feng, Polynomial splines over hierarchical T-meshes, Graphical Models 70 (2008) 76-86. doi : $10.1016 / j \cdot \operatorname{gmod} .2008 .03 .001$.

[14] T. Sederberg, J. Zheng, A. Bakenov, A. Nasri, T-splines and T-NURCCs,

[13] T. Dokken, T. Lyche, K. Pettersen, Polynomial splines over locally refined box partitions, Computer Aided Geometric Design 30 (2013) 331-356. doi : $10.1016 / \mathrm{j}$. cagd.2012.12.005

ACM Transactions on Graphics (TOG) 22 (2003) 477-484. doi:10.1145/ 882262.882295

[15] T. Sederberg, D. Cardon, G. Finnigan, N. North, J. Zheng, T. Lyche, Tspline simplification and local refinement, ACM Transactions on Graphics (TOG) 23 (2004) 276-283. doi:10.1145/1015706.1015715.

[16] M. Dörfel, B. Jüttler, B. Simeon, Adaptive isogeometric analysis by local h-refinenement with T-splines, Computer Methods in Applied Mechanics and Engineering 199 (2009) 264-275.

[17] J. Cottrell, T. H. Y. Bazilevs, Isogeometric analysis: Toward Integration of CAD and FEA, Wiley, Chichester, 2009.

[18] T. Hughes, J. Cottrell, Y. Bazilevs, Isogeometric analysis: CAD, finite elements, NURBS, exact geometry and mesh refinement, Computer Methods in Applied Mechanics and Engineering 194 (2005) 4135-4195.

[19] Y. Bazilevs, V. Calo, J. Cottrell, J. Evans, T. Hughes, S. Lipton, M. Scott, T. T. Sederberg, Isogeometric analysis using T-splines, Computer Methods 
in Applied Mechanics and Engineering 199 (2010) 229-263. doi:10.1007/ 978-3-7091-1843-6_5

[20] M. Scott, R. Simpson, J. Evans, S. Lipton, S. Bordas, T. Hughes, T. Sederberg, Isogeometric boundary element analysis using unstructured T-splines, Computer Methods in Applied Mechanics and Engineering 254 (2013) 177221. doi:10.1016/j.compstruc.2012.12.021.

[21] H. Lian, R. Simpson, S. Bordas, Sensitivity analysis and shape optimisation through a T-spline isogeometric boundary element method, in: International Conference on Computational Mechanics (CM13), Durham, UK, 2013.

[22] A.-A. Ginnis, K. Kostas, C. Politis, P. Kaklis, K. Belibassakis, T. Gerostathis, M. Scott, T. R. Hughes, Isogeometric boundary-element analysis for the wave-resistance problem using T-splines, Computer Methods in Applied Mechanics and Engineering 279 (2014) 425-439. doi: $10.1016 / \mathrm{j} . \mathrm{cma} .2014 .07 .001$.

[23] P. Kaklis, C. Politis, K. Belibassakis, A.-A. Ginnis, K. Kostas, T. Gerostathis, Boundary element methods and wave loading on ships, in: Encyclopedia of Computational Mechanics, 2nd Edition, ed. by Erwin Stein and René de Borst and Thomas J.R. Hughes, Chichester UK, John Wiley \& Sons, Ltd, 2018, pp. 1-35. doi:10.1002/9781119176817.ecm2115.

[24] X. Li, J. Zheng, T. Sederberg, T. Hughes, M. Scott, On the linear inde620 pendence of T-spline blending functions, Comput. Aided Geom. Design 29 (2012) 63-76.

[25] A. Bressan, A. Buffa, G. Sangalli, Characterisation of analysis-suitable Tsplines, Comput. Aided Geom. Design 39 (2015) 17-49.

[26] J. Zhang, X. Li, On degree-elevation of T-splines, Comput. Aided Geom. 
[27] MOERI, Moeri container ship (kcs) (2008).

URL http://www.simman2008.dk/KCS/container.html

[28] X. Li, M. A. Scott, Analysis-suitable T-splines: Characterization, refineability, and approximation, Mathematical Models and Methods in Applied Sciences 24 (2014) 1141-1164. doi:10.1142/S0218202513500796

[29] T. W. Sederberg, X. Li, H. Lin, H. Ipson, Watertight trimmed nurbs, ACM Transactions on Graphics (TOG) 27 (2008) 01-08. doi:10.1145/1360612. 1360678

[30] C. Hoffmann, K.-J. Kim, Towards valid parametric CAD modells, Computer-Aided Design 33 (2001) 81-90.

[31] S. Harries, C. Abt, M. Brenner, Upfront CAD - parametric modeling techniques for shape optimization, in: Advances in Evolutionary and Deterministic Methods for Design, Optimization and Control in Engineering and Sciences, Minisci E. et al. (Eds.), Springer 978-3-319-89986-2, Int. Conf. Evolutionary and Deterministic Methods for Design, Optimization and Control with Applications to Industrial and Societal Problems (EUROGEN), Glasgow, 2018, pp. 74-81.

[32] S. Harries, C. Abt, Parametric curve design applying fairness criteria, in: International Workshop on Creating Fair and Shape-Preserving Curves and Surfaces - Network Fairshape, (eds) H. Nowacki and P.D. Kaklis, Berlin, Potsdam, 1998. 